\title{
BIFURCATIONS OF WAVEFRONTS ON $r$-CORNERS: SEMI-LOCAL CLASSIFICATION*
}

\author{
TAKAHARU TSUKADA ${ }^{\dagger}$
}

\begin{abstract}
We introduce the notion of multi-reticular Legendrian unfoldings in order to investigate stabilities and a genericity of bifurcations of wavefronts generated by $m$ points of a hypersurface with a boundary, a corner, or an $r$-corner in a smooth $n$ dimensional manifold. We define several stabilities of multi-reticular Legendrian unfoldings and prove that they and the stabilities of corresponding generating families are all equivalent and give the classification of all generic bifurcations of their wavefronts in the cases $r=0, n \leq 5$ and $r=1, n \leq 3$ respectively.
\end{abstract}

Key words. Wavefront, bifurcation, Legendrian unfolding, $r$-corner.

AMS subject classifications. 58K25, 58K40, 53Dxx.

1. Introduction. In [3] K.Jänich explained the wavefront propagation mechanism on a manifold which is completely described by a positive and positively homogeneous Hamiltonian function on the cotangent bundle and investigated the local gradient models given by the ray length function. Caustics and Wavefronts generated by an initial wavefront which is a hypersurface germ without boundary in the manifold were investigated as Lagrangian and Legendrian singularities by V.I.Arnold (cf., $[1])$.

In this paper and its prequel [7], we investigate the stabilities and the genericity of bifurcations of wavefronts generated by a hypersurface germ with an r-corner. Wavefronts generated by all edges of the hypersurface germ at a time give a contact regular $r$-cubic configuration on the 1-jet bundle. All wavefronts around a time give a one-parameter family of contact regular $r$-cubic configurations on the 1 -jet bundle. In order to consider such families, we shall introduce the notion of unfolded contact regular $r$-cubic configurations on the big 1-jet bundle. A wavefront of an unfolded contact regular $r$-cubic configuration is the big front of the corresponding one-parameter family of contact regular $r$-cubic configurations. We shall consider their generating families and equivalence relations.

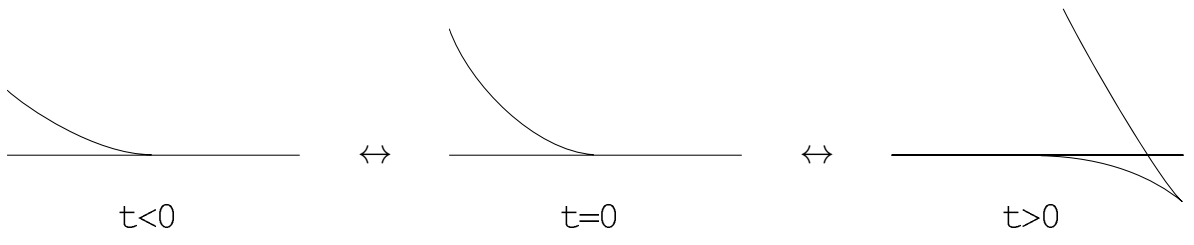

FIG. 1. The generic bifurcation of wavefronts on a boundary ${ }^{1} B_{3}$

We also consider bifurcations of intersections of wavefronts generated by $m$ points of the hypersurface. We introduce the notion of multi-reticular Legendrian unfoldings which is a generalized notion of multi-Legendrian unfoldings given by S.Izumiya (cf., [2]) for our situation. We shall define several stabilities of multi-reticular Legendrian

\footnotetext{
*Received December 3, 2010; accepted for publication March 2, 2012.

$\dagger$ College of Humanities \& Sciences, Department of Mathematics, Nihon University, Higashijujo 3-1-16 Kita-ku, Tokyo 114-0001, Japan (tsukada@math.chs.nihon-u.ac.jp).
} 
unfoldings and prove that they and the stabilities of their generating families are all equivalent. We shall also classify generic multi-reticular Legendrian unfoldings and give all figures of their wavefronts in the case $r=1, n \leq 3, m=1$ and $n \leq 3, m \geq 2$.

In history, our theory in the case $r=0$ is investigated as the theory of (multi)Legendrian unfoldings by S.Izumiya (cf., [2]) and the classification list of generic Legendrian unfoldings is given by using V.M.Zakalyukin's theory (cf., [10]) in which he has classified generic quasihomogeneous function germs under the $t$ - $\mathcal{P}$ - $\mathcal{K}$-equivalence. In [7] we have classified not only quasihomogeneous function germs but also all smooth function germs under the reticular $t-\mathcal{P}-\mathcal{K}$-equivalence which is a generalized relation of the $t$ - $\mathcal{P}$ - $\mathcal{K}$-equivalence.

I.G.Scherbak has studied the theory of boundary fronts in [4] and this corresponds to our theory in the case $r=m=1$. She has introduced the notion of Legendrian pairs which is corresponding to the notion of regular 1-cubic configurations. But they are not strictly formulated and no proof is given. In this paper, we shall define and prove our theory strictly. Since the equivalence relation of function germ used in her paper is slightly different from the one which is used in this paper (see the remark in Section 2), the figures of wavefronts ${ }^{0} B_{2}$ of fig. 2 and ${ }^{1} B_{3}$ of fig.3(cf., Figure 1), and ${ }^{0} B_{3}$ of fig. 4 in $[4$, p.365] do not coincide with our figures respectively. The classification list of function germs also different from ours (compare [4, p.371 Proposition 3] with [7, Theorem 4.7]).

For example we consider the below figures in which the initial wavefront with a boundary in a plane and wavefronts generated by the initial wavefront and the boundary to normal directions are described respectively. The generated wavefronts

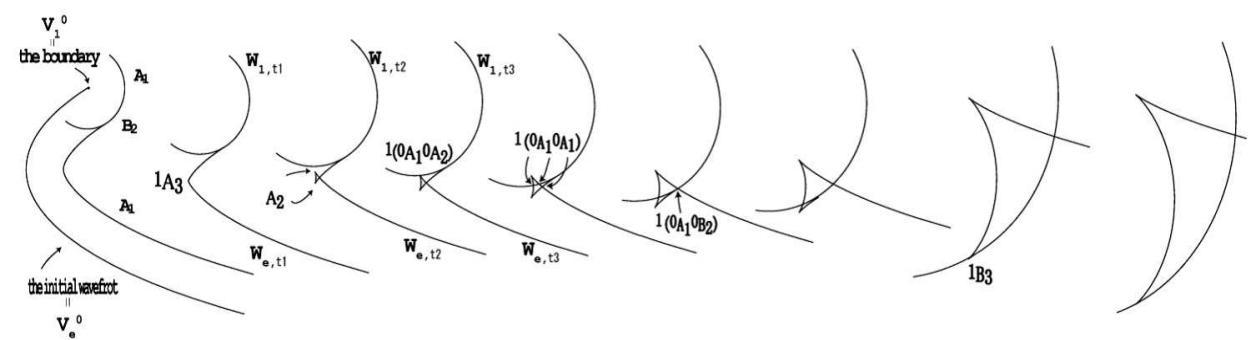

FIG. 2. The initial wavefront $V^{0}$ with a boundary and generated wavefronts $\left(e=\emptyset, t_{1}<t_{2}<t_{3}\right)$

bifurcate and typical shapes of bifurcations occur. The ${ }^{1} B_{3}$ front occurs (compare this to Figure 1$)$. The ${ }^{1}\left({ }^{0} A_{1}^{0} A_{2}\right),{ }^{1}\left({ }^{0} A_{1}{ }^{0} A_{1}\right)$, and ${ }^{1}\left({ }^{0} A_{1}{ }^{0} B_{2}\right)$ fronts are intersections of fronts generated by different points of the initial wavefront. The purpose of this paper is the classification of such generic bifurcations of wavefronts and their intersections.

This paper consists of six sections. In Section 2 we give the review of stabilities under the reticular $t-(\mathcal{P}-\mathcal{K})_{(m)}$-equivalence relation of map germs which play important roles as generating families of multi-reticular Legendrian unfoldings (cf., [7]). We shall also give the review of the theory of contact regular $r$-cubic configurations. In Section 3 we shall introduce the notion of multi-reticular Legendrian unfoldings and consider their generating families. In Section 4 we shall investigate several stabilities of multi-reticular Legendrian unfoldings. In Section 5 we shall reduce our investigation to finite dimensional jet spaces and give the classification of generic multi-reticular Legendrian unfoldings in the cases $r=0$ and 1 respectively. In Section 6 we shall 
show that our method do not work well for the cases $r \geq 2$ because of modalities of generating families. All maps considered here are differentiable of class $C^{\infty}$ unless stated otherwise.

2. Stabilities of unfoldings. In this section we investigate the theory of map germs with respect to the reticular $t-(\mathcal{P}-\mathcal{K})_{(m)}$-equivalence relation which is proved by almost parallel methods of [7].

Let $\mathbb{H}^{r}=\left\{\left(x_{1}, \ldots, x_{r}\right) \in \mathbb{R}^{r} \mid x_{1} \geq 0, \ldots, x_{r} \geq 0\right\}$ be an $r$-corner. We denote by $\mathcal{E}\left(r ; k_{1}, r ; k_{2}\right)$ the set of all germs at 0 of smooth maps $\mathbb{H}^{r} \times \mathbb{R}^{k_{1}} \rightarrow \mathbb{H}^{r} \times \mathbb{R}^{k_{2}}$ and set $\mathfrak{M}\left(r ; k_{1}, r ; k_{2}\right)=\left\{f \in \mathcal{E}\left(r ; k_{1}, r ; k_{2}\right) \mid f(0)=0\right\}$. We denote $\mathcal{E}\left(r ; k_{1}, k_{2}\right)$ for $\mathcal{E}\left(r ; k_{1}, 0 ; k_{2}\right)$ and denote $\mathfrak{M}\left(r ; k_{1}, k_{2}\right)$ for $\mathfrak{M}\left(r ; k_{1}, 0 ; k_{2}\right)$.

If $k_{2}=1$ we write simply $\mathcal{E}(r ; k)$ for $\mathcal{E}(r ; k, 1)$ and $\mathfrak{M}(r ; k)$ for $\mathfrak{M}(r ; k, 1)$. Then $\mathcal{E}(r ; k)$ is an $\mathbb{R}$-algebra in the usual way and $\mathfrak{M}(r ; k)$ is its unique maximal ideal. We also denote by $\mathcal{E}(k)$ for $\mathcal{E}(0 ; k)$ and $\mathfrak{M}(k)$ for $\mathfrak{M}(0 ; k)$. We remark that $\mathcal{E}(r ; k, p)$ is an $\mathcal{E}(r ; k)$-module generated by $p$-elements.

We denote by $J^{l}(r+k, p)$ the set of $l$-jets at 0 of germs in $\mathcal{E}(r ; k, p)$. There are natural projections:

$$
\pi_{l}: \mathcal{E}(r ; k, p) \longrightarrow J^{l}(r+k, p), \pi_{l_{2}}^{l_{1}}: J^{l_{1}}(r+k, p) \longrightarrow J^{l_{2}}(r+k, p)\left(l_{1}>l_{2}\right) .
$$

We write $j^{l} f(0)$ for $\pi_{l}(f)$ for each $f \in \mathcal{E}(r ; k, p)$.

Let $(x, y)=\left(x_{1}, \cdots, x_{r}, y_{1}, \cdots, y_{k}\right)$ be a fixed coordinate system of $\left(\mathbb{H}^{r} \times \mathbb{R}^{k}, 0\right)$. We denote by $\mathcal{B}(r ; k)$ the group of diffeomorphism germs $\left(\mathbb{H}^{r} \times \mathbb{R}^{k}, 0\right) \rightarrow\left(\mathbb{H}^{r} \times \mathbb{R}^{k}, 0\right)$ of the form:

$$
\phi(x, y)=\left(x_{1} \phi_{1}^{1}(x, y), \cdots, x_{r} \phi_{1}^{r}(x, y), \phi_{2}^{1}(x, y), \cdots, \phi_{2}^{k}(x, y)\right) .
$$

We denote by $\mathcal{B}_{n}(r ; k+n)$ the group of diffeomorphism germs $\left(\mathbb{H}^{r} \times \mathbb{R}^{k+n}, 0\right) \rightarrow$ $\left(\mathbb{H}^{r} \times \mathbb{R}^{k+n}, 0\right)$ of the form:

$$
\phi(x, y, u)=\left(x_{1} \phi_{1}^{1}(x, y, u), \cdots, x_{r} \phi_{1}^{r}(x, y, u), \phi_{2}^{1}(x, y, u), \cdots, \phi_{2}^{k}(x, y, u), \phi_{3}^{1}(u), \ldots, \phi_{3}^{n}(u)\right) .
$$

We denote $\phi(x, y, u)=\left(x \phi_{1}(x, y, u), \phi_{2}(x, y, u), \phi_{3}(u)\right), \frac{\partial f_{0}}{\partial y}=\left(\frac{\partial f_{0}}{\partial y_{1}}, \cdots, \frac{\partial f_{0}}{\partial y_{k}}\right)$, and denote other notations analogously.

Lemma 2.1. (cf., [9, Corollary 1.8]) Let $B$ be a submodule of $\mathcal{E}\left(r ; k+n+m^{\prime}, m\right)$, $A_{1}$ be a finitely generated $\mathcal{E}\left(m^{\prime}\right)$-submodule of $\mathcal{E}\left(r ; k+n+m^{\prime}, m\right)$ generated d-elements, and $A_{2}$ be a finitely generated $\mathcal{E}\left(n+m^{\prime}\right)$ submodule of $\mathcal{E}\left(r ; k+n+m^{\prime}, m\right)$. Suppose

$\mathcal{E}\left(r ; k+n+m^{\prime}, m\right)=B+A_{2}+A_{1}+\mathfrak{M}\left(m^{\prime}\right) \mathcal{E}\left(r ; k+n+m^{\prime}, m\right)+\mathfrak{M}\left(n+m^{\prime}\right)^{d+1} \mathcal{E}\left(r ; k+n+m^{\prime}, m\right)$.

Then

$$
\begin{gathered}
\mathcal{E}\left(r ; k+n+m^{\prime}, m\right)=B+A_{2}+A_{1}, \\
\mathfrak{M}\left(n+m^{\prime}\right)^{d} \mathcal{E}\left(r ; k+n+m^{\prime}, m\right) \subset B+A_{2}+\mathfrak{M}\left(m^{\prime}\right) \mathcal{E}\left(r ; k+n+m^{\prime}, m\right) .
\end{gathered}
$$

We say that $f_{0}=\left(f_{0,1}, \ldots, f_{0, m}\right)(x, y), g_{0}=\left(g_{0,1}, \ldots, g_{0, m}\right)(x, y) \in \mathcal{E}(r ; k, m)$ are reticular $\mathcal{K}_{(m)}$-equivalent if there exist $\Phi_{i} \in \mathcal{B}_{n}(r ; k+n)$ and units $a_{i} \in \mathcal{E}(r ; k+n)$ such that $g_{0, i}=a_{i} \cdot f_{0, i} \circ \Phi_{i}$ for $i=1, \ldots, m$. We denote $\Phi=\left(\Phi_{1}, \ldots, \Phi_{m}\right), a=\left(a_{1}, \ldots, a_{m}\right)$ and we call $(\Phi, a)$ a reticular $\mathcal{K}_{(m)}$-isomorphism from $f_{0}$ to $g_{0}$. We remark that $f_{0}$ and $g_{0}$ are reticular $\mathcal{K}_{(m)}$-equivalent if and only if $f_{0, i}$ and $g_{0, i}$ are reticular $\mathcal{K}$-equivalent 
for $i=1, \ldots, m$.

REMARK. The corresponding equivalence relation of function germs is given by I.G.Scherbak (see [4, p.366 §2]) as follows: Function germs $f_{0}, g_{0} \in \mathcal{E}(1+k)$ are equivalent if there exist a diffeomorphism germ $\phi$ on $\left(\mathbb{R}^{1+k}, 0\right)$ of the form $\phi(x, y)=$ $\left(x \phi_{1}(x, y), \phi_{2}^{1}(x, y), \ldots, \phi_{2}^{k}(x, y)\right)$ and a unit $a \in \mathcal{E}(r ; k)$ such that $g_{0}=a \cdot f_{0} \circ \phi$. The variable $x$ is defined on $(\mathbb{H}, 0)$ and $\phi_{1}(0)>0$ in our equivalence relation. On the other hand $x$ is defined on $(\mathbb{R}, 0)$ and the condition $\phi_{1}(0)>0$ is not required in I.G.Scherbak's equivalence relation. These differences appear in the definition of wavefronts and consequently the figures of wavefronts in [4] and the figures in this paper are different to each other.

LEMMA 2.2. Let $f_{0}(x, y) \in \mathfrak{M}(r ; k, m)$ and $z=j^{l} f_{0}(0)$. Let $O_{r \mathcal{K}}^{l}(z)$ be the submanifold of $J^{l}(r+k, m)$ consist of the image by $\pi_{l}$ of the orbit under the reticular $\mathcal{K}_{(m)}$-equivalence of $f_{0}$. Put $z=j^{l} f_{0}(0)$. Then

$$
\begin{aligned}
T_{z}\left(O_{r \mathcal{K}}^{l}(z)\right)=\pi_{l}( & \left(\left\langle f_{0,1}, x \frac{\partial f_{0,1}}{\partial x}\right\rangle_{\mathcal{E}(r ; k)}+\mathfrak{M}(r ; k)\left\langle\frac{\partial f_{0,1}}{\partial y}\right\rangle\right) \\
& \left.\times \cdots \times\left(\left\langle f_{0, m}, x \frac{\partial f_{0, m}}{\partial x}\right\rangle_{\mathcal{E}(r ; k)}+\mathfrak{M}(r ; k)\left\langle\frac{\partial f_{0, m}}{\partial y}\right\rangle\right)\right)
\end{aligned}
$$

We say that a map germ $f_{0}=\left(f_{0,1}, \ldots, f_{0, m}\right)(x, y) \in \mathfrak{M}(r ; k, m)$ is reticular $\mathcal{K}_{(m)}$ l-determined if all map germ in $\mathfrak{M}(r ; k, m)$ which has the same $l$-jet of $f_{0}$ is reticular $\mathcal{K}_{(m)}$-equivalent to $f_{0}$.

Lemma 2.3. (cf., $\left[7\right.$, Lemma 2.3]) Let $f_{0}=\left(f_{0,1}, \ldots, f_{0, m}\right)(x, y) \in \mathfrak{M}(r ; k, m)$ and let

$$
\mathfrak{M}(r ; k)^{l+1} \subset \mathfrak{M}(r ; k)\left(\left\langle f_{0, i}, x \frac{\partial f_{0, i}}{\partial x_{1}}\right\rangle+\mathfrak{M}(r ; k)\left\langle\frac{\partial f_{0, i}}{\partial y}\right\rangle\right)+\mathfrak{M}(r ; k)^{l+2}
$$

for $i=1, \ldots, m$. Then $f_{0}$ is reticular $\mathcal{K}_{(m)}$-l-determined. Conversely if $f_{0}(x, y) \in$ $\mathfrak{M}(r ; k, m)$ is reticular $\mathcal{K}_{(m)}$-l-determined, then

$$
\mathfrak{M}(r ; k)^{l+1} \subset\left\langle f_{0, i}, x \frac{\partial f_{0, i}}{\partial x_{1}}\right\rangle_{\mathcal{E}(r ; k)}+\mathfrak{M}(r ; k)\left\langle\frac{\partial f_{0, i}}{\partial y}\right\rangle \text { for } i=1, \ldots, m .
$$

We say that $f=\left(f_{1}, \ldots, f_{m}\right)(x, y, u), g=\left(g_{1}, \ldots, g_{m}\right)(x, y, u) \in \mathcal{E}(r ; k+n, m)$ are reticular $(\mathcal{P}-\mathcal{K})_{(m)}$-equivalent if there exist $\Phi_{i} \in \mathcal{B}_{n}(r ; k+n)$ of the form:

$$
\Phi_{i}(x, y, u)=\left(x \phi_{1}^{i}(x, y, t, u), \phi_{2}^{i}(x, y, t, u), \phi_{3}(u)\right)
$$

and a unit $a=\left(a_{1}, \ldots, a_{m}\right)(x, y, u) \in \mathcal{E}(r ; k+n, m)$ such that $g_{i}=a_{i} \cdot f_{i} \circ \Phi_{i}$ for $i=1, \ldots, m$. We write $\Phi=\left(\Phi_{1}, \ldots, \Phi_{m}\right)$ and call $(\Phi, a)$ a reticular $(\mathcal{P}-\mathcal{K})_{(m)^{-}}$ isomorphism from $f$ to $g$.

We say that a map germ $f=\left(f_{1}, \ldots, f_{m}\right)(x, y, u) \in \mathfrak{M}(r ; k+n, m)$ is reticular $(\mathcal{P}-\mathcal{K})_{(m)}$-l-determined if all map germ in $\mathfrak{M}(r ; k+n, m)$ which has the same $l$-jet of $f$ is reticular $(\mathcal{P}-\mathcal{K})_{(m)}$-equivalent to $f$. 
For $f=\left(f_{1}, \ldots, f_{m}\right)(x, y, u) \in \mathcal{E}(r ; k+n, m)$ we set

$$
\begin{array}{r}
O_{r \mathcal{P}-\mathcal{K}_{(m)}}(f)=\left(\left\langle f_{1}, x \frac{\partial f_{1}}{\partial x}\right\rangle_{\mathcal{E}(r ; k+n)}+\mathfrak{M}(r ; k+n)\left\langle\frac{\partial f_{1}}{\partial y}\right\rangle\right) \times \cdots \times\left(\left\langle f_{m}, x \frac{\partial f_{m}}{\partial x}\right\rangle_{\mathcal{E}(r ; k+n)}\right. \\
\left.+\mathfrak{M}(r ; k+n)\left\langle\frac{\partial f_{m}}{\partial y}\right\rangle\right)+\mathfrak{M}(n)\left\langle\frac{\partial f}{\partial u}\right\rangle .
\end{array}
$$

LEMMA 2.4. Let $f=\left(f_{1}, \ldots, f_{m}\right)(x, y, u) \in \mathcal{E}(r ; k+n, m)$ and $z=j^{l} f(0)$. We write $O_{r \mathcal{P}-\mathcal{K}_{(m)}}^{l}(z)$ the submanifold which consists of l-jets of the orbit of $f$ under the reticular $t-(\mathcal{P}-\mathcal{K})_{(m)}$ equivalence relation. Then it holds that

$$
O_{r \mathcal{P}-\mathcal{K}_{(m)}}^{l}(z)=\pi_{l}\left(O_{r \mathcal{P}-\mathcal{K}_{(m)}}(f)\right)
$$

Lemma 2.5. (cf., [7, Lemma 3.10]) Let $f=\left(f_{1}, \ldots, f_{m}\right)(x, y, u) \in \mathfrak{M}(r ; k+n, m)$ and $l$ be a non-negative integer. If

$$
\mathfrak{M}(r ; k+n, m)^{l} \subset O_{r \mathcal{P}-\mathcal{K}_{(m)}}(f)+\mathfrak{M}(n) \mathfrak{M}(r ; k+n, m)^{l},
$$

then $f$ is reticular $(\mathcal{P}-\mathcal{K})_{(m)}$-l-determined.

In convenience, we denote an unfolding of a function germ $f(x, y, u) \in \mathfrak{M}(r ; k+$ $n, m)$ by $F(x, y, t, u) \in \mathfrak{M}\left(r ; k+m^{\prime}+n, m\right)$.

Let $F(x, y, t, u) \in \mathfrak{M}\left(r ; k+m_{1}^{\prime}+n, m\right)$ and $G(x, y, s, u) \in \mathfrak{M}\left(r ; k+m_{2}^{\prime}+n, m\right)$ be unfoldings of $f(x, y, u) \in \mathfrak{M}(r ; k+n, m)$.

$A$ reticular $t-(\mathcal{P}-\mathcal{K})_{(m)}-f$-morphism from $F$ to $G$ is a pair $(\Phi, a)$, where $\Phi=$ $\left(\Phi_{1}, \ldots, \Phi_{m}\right)$ for $\Phi_{i} \in \mathfrak{M}\left(r ; k+m_{2}^{\prime}+n, r ; k+m_{1}^{\prime}+n\right)$ and $a=\left(a_{1}, \ldots, a_{m}\right)$ is a unit of $\mathcal{E}\left(r ; k+m_{2}^{\prime}+n, m\right)$ satisfying the following conditions:

(1) $\Phi_{i}$ can be written in the form:

$$
\Phi_{i}(x, y, t, u)=\left(x \phi_{1}^{i}(x, y, t, u), \phi_{2}^{i}(x, y, t, u), \phi_{3}(t), \phi_{4}(t, u)\right),
$$

(2) $\left.\Phi_{i}\right|_{\mathbb{H}^{r} \times \mathbb{R}^{k+n}}=i d_{\mathbb{H}^{r} \times \mathbb{R}^{k+n}},\left.a_{i}\right|_{\mathbb{H}^{r} \times \mathbb{R}^{k+n}} \equiv 1$,

(3) $G_{i}(x, y, t, u)=a_{i}(x, y, t, u) \cdot F_{i} \circ \Phi_{i}(x, y, t, u)$ for all $(x, y, t, u) \in\left(\mathbb{H}^{r} \times \mathbb{R}^{k+m_{2}^{\prime}+n}, 0\right)$.

If there exists a reticular $t$ - $(\mathcal{P}-\mathcal{K})_{(m)}-f$-morphism from $F$ to $G$, we say that $G$ is reticular $t-(\mathcal{P}-\mathcal{K})_{(m)}-f$-induced from $F$. If $m_{1}=m_{2}$ and $\Phi$ is invertible, we call $(\Phi, a)$ a reticular $t-(\mathcal{P}-\mathcal{K})_{(m)}-f$-isomorphism from $F$ to $G$ and we say that $F$ is reticular $t$ - $(\mathcal{P}-\mathcal{K})_{(m)}-f$-equivalent to $G$.

We say that $F(x, y, t, u), G(x, y, t, u) \in \mathcal{E}\left(r ; k+m^{\prime}+n, m\right)$ are reticular $t$ - $(\mathcal{P}$ $\mathcal{K})_{(m)}$-equivalent if there exist diffeomorphism germs

$$
\Phi_{i}:\left(\mathbb{H}^{r} \times \mathbb{R}^{k+m^{\prime}+n}, 0\right) \rightarrow\left(\mathbb{H}^{r} \times \mathbb{R}^{k+m^{\prime}+n}, 0\right) \quad(i=1, \ldots, m)
$$

of the form

$$
\Phi_{i}(x, y, t, q, z)=\left(x \phi_{1}^{i}(x, y, t, u), \phi_{2}^{i}(x, y, t, u), \phi_{3}(t), \phi_{4}(t, u)\right)
$$

and a unit $a=\left(a_{1}, \ldots, a_{m}\right) \in \mathcal{E}\left(r ; k+m^{\prime}+n, m\right)$ such that $G_{i}=a_{i} \cdot F_{i} \circ \Phi_{i}$ for $i=1, \ldots, m$. We call $(\Phi, a)$ a reticular $t-(\mathcal{P}-\mathcal{K})_{(m)}$-isomorphism from $F$ to $G$. 
We say that function germs $F_{1}\left(x_{1}, \ldots, x_{r_{1}}, y_{1}, \cdots, y_{k_{1}}, t, q, z\right) \in \mathfrak{M}\left(r_{1} ; k_{1}+m^{\prime}+\right.$ $n+1)$ and $F_{2}\left(x_{1}, \ldots, x_{r_{2}}, y_{1}, \cdots, y_{k_{2}}, t, q, z\right) \in \mathfrak{M}\left(r_{2} ; k_{2}+m^{\prime}+n+1\right)$ are stably reticular $t$-P $\mathcal{P}-\mathcal{K}$-equivalent if $F_{1}$ and $F_{2}$ are reticular $t$ - $\mathcal{P}-\mathcal{K}$-equivalent after additions of linear forms of $x$ of which all coefficients are not zero and non-degenerate quadratic forms in the variables $y$.

We define the notion of stable reticular $t-(\mathcal{P}-\mathcal{K})_{(m)}$-equivalence in the same method of the stable reticular $t$ - $\mathcal{P}$ - $\mathcal{K}$-equivalence.

Definition 2.6. We define stabilities of unfoldings. Let $F=\left(F_{1}\right.$, $\left.\ldots, F_{m}\right)(x, y, t, u) \in \mathfrak{M}\left(r ; k+m^{\prime}+n, m\right)$ be an unfolding of $f=\left(f_{1}\right.$, $\left.\ldots, f_{m}\right)(x, y, u) \in \mathfrak{M}(r ; k+n, m)$.

We say that $F$ is reticular $t-(\mathcal{P}-\mathcal{K})_{(m)}$-stable if the following condition holds: For any neighborhood $U$ of 0 in $\mathbb{H}^{r} \times \mathbb{R}^{k+m^{\prime}+n}$ and any representative $\tilde{F} \in C^{\infty}\left(U, \mathbb{R}^{m}\right)$, there exists a neighborhood $N_{\tilde{F}}$ of $\tilde{F}$ in $C^{\infty}$-topology such that for any $\tilde{G} \in N_{\tilde{F}}$ there exist $\left(0, y^{i}, t^{0}, u^{0}\right) \in U$ for $i=1, \ldots, m$ such that $G=\left(G_{1}, \ldots, G_{m}\right)$ and $F$ are reticular $t$ - $(\mathcal{P}-\mathcal{K})_{(m)}$-equivalent, where $G_{i} \in \mathfrak{M}\left(r ; k+m^{\prime}+n\right)$ is defined by $G_{i}(x, y, t, u)=\tilde{G}\left(x, y+y^{i}, t+t^{0}, u+u^{0}\right)-\tilde{G}\left(0, y^{i}, t^{0}, u^{0}\right)$.

We say that $F$ is a reticular $t-(\mathcal{P}-\mathcal{K})_{(m)}$-versal unfolding of $f$ if any unfolding of $f$ is reticular $t-(\mathcal{P}-\mathcal{K})_{(m)^{-}} f$-induced from $F$. We say that $F$ is a reticular $t-(\mathcal{P}-\mathcal{K})_{(m)^{-}}$ universal unfolding of $f$ if $m$ is minimal in reticular $t$ - $(\mathcal{P}-\mathcal{K})_{(m)}$-versal unfoldings of $f$.

We say that $F$ is reticular $t-(\mathcal{P}-\mathcal{K})_{(m)}$-infinitesimally versal if

$$
\begin{aligned}
& \mathcal{E}(r ; k+n, m)=\left\langle f_{1}, x \frac{\partial f_{1}}{\partial x}, \frac{\partial f_{1}}{\partial y}\right\rangle_{\mathcal{E}(r ; k+n)} \times \cdots \times \\
&\left\langle f_{m}, x \frac{\partial f_{m}}{\partial x}, \frac{\partial f_{m}}{\partial y}\right\rangle_{\mathcal{E}(r ; k+n)}+\left\langle\frac{\partial f}{\partial u}\right\rangle_{\mathcal{E}(n)}+\left\langle\left.\frac{\partial F}{\partial t}\right|_{t=0}\right\rangle_{\mathbb{R}}
\end{aligned}
$$

We say that $F$ is reticular $t-(\mathcal{P}-\mathcal{K})_{(m)}$-infinitesimally stable if

$$
\begin{aligned}
& \mathcal{E}\left(r ; k+m^{\prime}+\right.n, m)=\left\langle F_{1}, x \frac{\partial F_{1}}{\partial x}, \frac{\partial F_{1}}{\partial y}\right\rangle_{\mathcal{E}\left(r ; k+m^{\prime}+n\right)} \times \cdots \times \\
&\left\langle F_{m}, x \frac{\partial F_{m}}{\partial x}, \frac{\partial F_{m}}{\partial y}\right\rangle_{\mathcal{E}\left(r ; k+m^{\prime}+n\right)}+\left\langle\frac{\partial F}{\partial u}\right\rangle_{\mathcal{E}\left(m^{\prime}+n\right)}+\left\langle\frac{\partial F}{\partial t}\right\rangle_{\mathcal{E}\left(m^{\prime}\right)}
\end{aligned}
$$

We say that $F$ is reticular $t-(\mathcal{P}-\mathcal{K})_{(m)}$-homotopically stable if for any smooth pathgerm $(\mathbb{R}, 0) \rightarrow \mathcal{E}\left(r ; k+m^{\prime}+n, m\right), \tau \mapsto F_{\tau}=\left(F_{1, \tau}, \ldots, F_{m, \tau}\right)$ with $F_{0}=F$, there exists a smooth path-germs $(\mathbb{R}, 0) \rightarrow \mathcal{B}\left(r ; k+m^{\prime}+n\right) \times \mathcal{E}\left(r ; k+m^{\prime}+n\right), \tau \mapsto\left(\Phi_{\tau}^{i}, a_{\tau}^{i}\right)$ with $\left(\Phi_{0}^{i}, a_{0}^{i}\right)=(i d, 1)$ and $\Phi_{\tau}^{i}$ has the form

$$
\Phi_{\tau}^{i}(x, y, t, u)=\left(x \phi_{\tau}^{i, 1}(x, y, t, u), \phi_{\tau}^{i, 2}(x, y, t, u), \phi_{\tau}^{3}(t), \phi_{\tau}^{4}(t, u)\right)
$$

such that each $\left(\Phi_{\tau}^{i}, a_{\tau}^{i}\right)$ is a reticular $t$ - $\mathcal{P}$ - $\mathcal{K}$-isomorphism and $F_{i, \tau}=a_{\tau}^{i} \cdot F_{i, 0} \circ \Phi_{\tau}^{i}$ for $\tau \in(\mathbb{R}, 0)$ and $i=1, \ldots, m$.

Let $U$ be a neighborhood of 0 in $\mathbb{H}^{r} \times \mathbb{R}^{k+m^{\prime}+n}, \tilde{F}=\left(\tilde{F}^{1}, \ldots, \tilde{F}^{m}\right): U \rightarrow \mathbb{R}^{m}$ be a smooth map, and $l$ be a non-negative integer. We choose a neighborhood $U^{\prime}$ of 0 in 
$\mathbb{R}^{k m+m^{\prime}+n}$ such that $\left(0, y^{i}, t, u\right) \in U$ for any $\left(y^{1}, \ldots, y^{m}, t, u\right) \in U^{\prime}$ and $i$. We define the smooth map germ

$$
j_{1}^{l} \tilde{F}: U^{\prime} \longrightarrow J^{l}(r+k+n, m)
$$

as follows: For $\left(y^{1}, \ldots, y^{m}, t, u\right) \in U$ we set $j_{1}^{l} \tilde{F}\left(y^{1}, \ldots, y^{m}, t, u\right)$ by the $l$-jet of the map germ $\left(f_{\left(y^{1}, t, u\right)}^{1}, \ldots, f_{\left(y^{m}, t, u\right)}^{m}\right) \in \mathfrak{M}(r ; k+n, m)$ at 0 , where $f_{\left(y^{i}, t, u\right)}^{i}$ is given by $f_{\left(y^{i}, t, u\right)}^{i}\left(x^{\prime}, y^{\prime}, u^{\prime}\right)=\tilde{F}^{i}\left(x^{\prime}, y^{i}+y^{\prime}, t, u+u^{\prime}\right)-\tilde{F}^{i}\left(0, y^{i}, t, u\right)$

Let $F(x, y, t, u) \in \mathfrak{M}\left(r ; k+m^{\prime}+n, m\right)$ be an unfolding of $f(x, y, u) \in \mathfrak{M}(r ; k+$ $n, m)$. Let $l$ be a non-negative integer and $z=j^{l} f(0)$. We say that $F$ is reticular $t$ - $(\mathcal{P}-\mathcal{K})_{(m)}-l$-transversal if $j_{1}^{l} \tilde{F}$ at 0 is transversal to $O_{r \mathcal{P}-\mathcal{K}_{(m)}}^{l}(z)$ for a representative $\tilde{F} \in C^{\infty}\left(U, \mathbb{R}^{m}\right)$ of $F$.

Lemma 2.7. (cf., [7, Lemma 3.4]) Let $F(x, y, t, u) \in \mathfrak{M}\left(r ; k+m^{\prime}+n, m\right)$ be an unfolding of $f(x, y, u) \in \mathfrak{M}(r ; k+n, m)$. Then $F$ is reticular $t-(\mathcal{P}-\mathcal{K})_{(m)}$-l-transversal unfolding of $f$ if and only if

$$
\begin{aligned}
\mathcal{E}(r ; k+n, m)= & \left\langle f_{1}, x \frac{\partial f_{1}}{\partial x}, \frac{\partial f_{1}}{\partial y}\right\rangle_{\mathcal{E}(r ; k+n)} \times \cdots \times\left\langle f_{m}, x \frac{\partial f_{m}}{\partial x}, \frac{\partial f_{m}}{\partial y}\right\rangle_{\mathcal{E}(r ; k+n)} \\
& +\left\langle\frac{\partial f}{\partial u}\right\rangle_{\mathcal{E}(n)}+\left\langle\left.\frac{\partial F}{\partial t}\right|_{t=0}\right\rangle_{\mathbb{R}}+\mathfrak{M}(r ; k+n)^{l+1} \mathcal{E}(r ; k+n, m) .
\end{aligned}
$$

Theorem 2.8. (Uniqueness of universal unfoldings) (cf., [7, Theorem 3.13]) Let $F(x, y, u, t), G(x, y, u, t) \in \mathfrak{M}\left(r ; k+n+m^{\prime}, m\right)$ be unfoldings of $f \in \mathfrak{M}(r ; k+n, m)$. If $F$ and $G$ are reticular $t-(\mathcal{P}-\mathcal{K})_{(m)}$-universal, then $F$ and $G$ are reticular $t-\mathcal{P}-\mathcal{K}-f$ equivalent.

The following theorem is used to prove Theorem 4.6.

Theorem 2.9. (cf., [7, Theorem 3.14]) Let $F(x, y, t, u) \in \mathfrak{M}\left(r ; k+m^{\prime}+n, m\right)$ be an unfolding of $f(x, y, u) \in \mathfrak{M}(r ; k+n, m)$ and let $f$ is an unfolding of $f_{0}(x, y) \in$ $\mathfrak{M}(r ; k, m)$. Then following are equivalent.

(1) There exists a non-negative number $l$ such that $f_{0}$ is reticular $\mathcal{K}_{(m)}$-l-determined and $F$ is reticular $t-(\mathcal{P}-\mathcal{K})_{(m)}-l^{\prime}$-transversal for $l^{\prime} \geq l m+l+m^{\prime}$,

(2) $F$ is reticular $t-(\mathcal{P}-\mathcal{K})_{(m)}$-stable,

(3) $F$ is reticular $t-(\mathcal{P}-\mathcal{K})_{(m)}$-versal,

(4) $F$ is reticular $t-(\mathcal{P}-\mathcal{K})_{(m)}$-infinitesimally versal,

(5) $F$ is reticular $t-(\mathcal{P}-\mathcal{K})_{(m)}$-infinitesimally stable,

(6) $F$ is reticular $t-(\mathcal{P}-\mathcal{K})_{(m)}$-homotopically stable.

3. Reticular Legendrian unfoldings. In order to consider one-parameter families of Legendrian submanifold with an $r$-corner, we require the notion of reticular Legendrian unfoldings, their equivalence relation, and generating families.

We consider the big 1-jet bundle $J^{1}\left(\mathbb{R} \times \mathbb{R}^{n}, \mathbb{R}\right)$ and the canonical 1-form $\Theta$ on that space. Let $(t, q)=\left(t, q_{1}, \ldots, q_{n}\right)$ be the canonical coordinate system on $\mathbb{R} \times \mathbb{R}^{n}$ and $(t, q, z, s, p)=\left(t, q_{1}, \ldots, q_{n}, z, s, p_{1}, \ldots, p_{n}\right)$ be the corresponding coordinate system on $J^{1}\left(\mathbb{R} \times \mathbb{R}^{n}, \mathbb{R}\right)$. Then the canonical 1-form $\Theta$ is given by

$$
\Theta=d z-\sum_{i=1}^{n} p_{i} d q_{i}-s d t=\theta-s d t
$$


where $\theta$ is the canonical 1-form on $J^{1}\left(\mathbb{R}^{n}, \mathbb{R}\right)$.

Let $\left\{L_{\sigma, t}\right\}_{\sigma \subset I_{r}, t \in(\mathbb{R}, 0)}$ be a family of contact regular $r$-cubic configurations on $J^{1}\left(\mathbb{R}^{n}, \mathbb{R}\right)$ defined by a family of contact embedding germs $C_{t}:\left(J^{1}\left(\mathbb{R}^{n}, \mathbb{R}\right), 0\right) \rightarrow$ $J^{1}\left(\mathbb{R}^{n}, \mathbb{R}\right)$ depending smoothly on $t \in(\mathbb{R}, 0)$ such that $\pi \circ C_{0}(0)=0$ and $L_{\sigma, t}=C_{t}\left(L_{\sigma}^{0}\right)$ for all $\sigma \subset I_{r}$ and $t \in(\mathbb{R}, 0)$.

Then we consider the following contact diffeomorphism germ $C$ on $\left(J^{1}(\mathbb{R} \times\right.$ $\left.\left.\mathbb{R}^{n}, \mathbb{R}\right), w\right)(\Pi(w)=0)$ :

Lemma 3.1. For any family of contact embedding germs $C_{t}:\left(J^{1}\left(\mathbb{R}^{n}, \mathbb{R}\right), 0\right) \rightarrow$ $J^{1}\left(\mathbb{R}^{n}, \mathbb{R}\right)\left(\pi \circ C_{0}(0)=0\right)$ depending smoothly on $t \in(\mathbb{R}, 0)$, there exists a unique function germ $h$ on $\left(J^{1}\left(\mathbb{R} \times \mathbb{R}^{n}, \mathbb{R}\right), 0\right)$ such that the map germ $C:\left(J^{1}\left(\mathbb{R} \times \mathbb{R}^{n}, \mathbb{R}\right), 0\right) \rightarrow$ $\left(J^{1}\left(\mathbb{R} \times \mathbb{R}^{n}, \mathbb{R}\right), 0\right)$ defined by

$$
C(t, q, z, s, p)=\left(t, q \circ C_{t}(q, z, p), z \circ C_{t}(q, z, p), h(t, q, z, s, p), p \circ C_{t}(q, z, p)\right)
$$

is a contact diffeomorphism.

Proof. We denote $C_{t}(q, z, p)=\left(q_{t}(q, z, p), z_{t}(z, q, p), p_{t}(q, z, p)\right)$. Since $C_{t}$ is a contact embedding germ for all $t \in(\mathbb{R}, 0)$, there exists a function germ $\alpha(t, q, z, p)$ around zero with $\alpha(0) \neq 0$ such that $d z_{t}(q, z, p)-p_{t}(q, z, p) d q_{t}(q, z, p)=\alpha(t, q, z, p)(d z-p d q)$ for all fixed t. By the direct calculation of this equation, we have that

$$
\frac{\partial z_{t}}{\partial z}-p_{t} \frac{\partial q_{t}}{\partial z}=\alpha, \frac{\partial z_{t}}{\partial q}-p_{t} \frac{\partial q_{t}}{\partial q}=-p \alpha, \frac{\partial z_{t}}{\partial p}-p_{t} \frac{\partial q_{t}}{\partial p}=0 .
$$

We also calculate $C^{*}(d z-p d q-s d t)$ by considering the above relations. Then we have that

$$
\begin{aligned}
& C^{*}(d z-p d q-s d t) \\
= & d z_{t}(z, q, p)-p_{t}(q, z, p) d q_{t}(q, z, p)-h(t, q, z, s, p) d t \\
= & \alpha(t, z, q, p) d z-\alpha(t, z, q, p) p d q-\left(\frac{\partial z_{t}}{\partial t}(q, z, p)-p_{t}(q, z, p) \frac{\partial q_{t}}{\partial t}(q, z, p)-h(t, q, z, s, p)\right) d t .
\end{aligned}
$$

In order to make $C$ a contact embedding, the function $h(t, q, z, s, p)$ is uniquely determined that:

$$
h(t, q, z, s, p)=\frac{\partial z_{t}}{\partial t}(q, z, p)-p_{t}(q, z, p) \frac{\partial q_{t}}{\partial t}(q, z, p)+\alpha(t, q, z, p) s .
$$

Definition 3.2. Let $C$ be a contact embedding germ $\left(J^{1}\left(\mathbb{R} \times \mathbb{R}^{n}, \mathbb{R}\right), 0\right) \rightarrow$ $J^{1}\left(\mathbb{R} \times \mathbb{R}^{n}, \mathbb{R}\right)$. We say that $C$ is a $\mathcal{P}$-contact diffeomorphism if $C$ has the form:

$$
C(t, q, z, s, p)=\left(t, q_{C}(t, q, z, p), z_{C}(t, q, z, p), h_{C}(t, q, z, s, p), p_{C}(t, q, z, p)\right) .
$$

We remark that a $\mathcal{P}$-contact diffeomorphism and the corresponding oneparameter family of contact embedding germs are uniquely defined by each other.

We define that $\tilde{L}_{\sigma}^{r, 0}=\left\{(t, q, z, s, p) \in J^{1}\left(\mathbb{R} \times \mathbb{R}^{n}, \mathbb{R}\right) \mid q_{\sigma}=p_{I_{r}-\sigma}=q_{r+1}=\cdots=\right.$ $\left.q_{n}=s=z=0, q_{I_{r}-\sigma} \geq 0\right\}$ for $\sigma \subset I_{r}$ and $\mathbb{L}^{r}=\left\{(t, q, z, s, p) \in J^{1}\left(\mathbb{R} \times \mathbb{R}^{n}, \mathbb{R}\right) \mid q_{1} p_{1}=\right.$ $\left.\cdots=q_{r} p_{r}=q_{r+1}=\cdots=q_{n}=s=z=0, q_{I_{r}} \geq 0\right\}$ be a representative as a germ of the union of $\tilde{L}_{\sigma}^{r, 0}$ for all $\sigma \subset I_{r}$. We denote that the set $\tilde{L}_{\sigma}^{r, 0}$ is the normalization of the particles incident from the $\sigma$-corner of the initial hypersurface germ with a $r$-corner at time $t$. 
Definition 3.3. We say that a map germ $\mathcal{L}:\left(\mathbb{L}^{r}, 0\right) \rightarrow\left(J^{1}(\mathbb{R} \times\right.$ $\left.\left.\mathbb{R}^{n}, \mathbb{R}\right), w\right)(\Pi(w)=0)$ is a reticular Legendrian unfolding if $\mathcal{L}$ is the restriction of a $\mathcal{P}$-contact diffeomorphism. We call $\left\{\mathcal{L}\left(\tilde{L}_{\sigma}^{r, 0}\right)\right\}_{\sigma \subset I_{r}}$ the unfolded contact regular $r$ cubic configuration of $\mathcal{L}$.

We note that: Let $\left\{\tilde{L}_{\sigma}\right\}_{\sigma \subset I_{r}}$ be an unfolded contact regular $r$-cubic configuration associated with a one-parameter family of contact regular $r$-cubic configurations $\left\{L_{\sigma, t}\right\}_{\sigma \subset I_{r}, t \in(\mathbb{R}, 0)}$. Then there is the following relation between the wavefront $W_{\sigma}=\Pi\left(\tilde{L}_{\sigma}\right)$ and the family of wavefronts $W_{\sigma, t}=\pi\left(L_{\sigma, t}\right)$ :

$$
W_{\sigma}=\bigcup_{t \in(\mathbb{R}, 0)}\{t\} \times W_{\sigma, t} \quad \text { for all } \sigma \subset I_{r}
$$

In order to study bifurcations of wavefronts of unfolded contact regular $r$-cubic configurations we introduce the following equivalence relation. Let $\Psi$ be a contact diffeomorphism germ on $\left(J^{1}\left(\mathbb{R} \times \mathbb{R}^{n}, \mathbb{R}\right), 0\right)$. We say that $\Psi$ is a reticular $\mathcal{P}_{(r)^{-}}$ diffeomorphism if $\pi_{t} \circ \Psi$ depends only on $t$ and $\Psi$ preserves $\tilde{L}_{\sigma}^{r, 0}$ for all $\sigma \subset I_{r}$.

Let $\left\{\tilde{L}_{\sigma}^{i}\right\}_{\sigma \subset I_{r}}(i=1,2)$ be unfolded contact regular $r$-cubic configurations on $\left(J^{1}\left(\mathbb{R} \times \mathbb{R}^{n}, \mathbb{R}\right), w_{i}\right)\left(\Pi\left(w_{i}\right)=0\right)$. We say that a contact diffeomorphism germ $K$ : $\left(J^{1}\left(\mathbb{R} \times \mathbb{R}^{n}, \mathbb{R}\right), w_{1}\right) \rightarrow\left(J^{1}\left(\mathbb{R} \times \mathbb{R}^{n}, \mathbb{R}\right), w_{2}\right)$ is a $\mathcal{P}$-Legendrian equivalence if $K$ has the form:

$$
K(t, q, z, s, p)=\left(\phi_{1}(t), \phi_{2}(t, q, z), \phi_{3}(t, q, z), \phi_{4}(t, q, z, s, p), \phi_{5}(t, q, z, s, p)\right) .
$$

We say that they are $\mathcal{P}$-Legendrian equivalent if there exist a $\mathcal{P}$-Legendrian equivalence $K$ such that $\tilde{L}_{\sigma}^{2}=K\left(\tilde{L}_{\sigma}^{1}\right)$ for all $\sigma \subset I_{r}$.

In order to understand the meaning of $\mathcal{P}$-Legendrian equivalence, we observe the following: Let $\left\{\tilde{L}_{\sigma}^{i}\right\}_{\sigma \subset I_{r}}(i=1,2)$ be unfolded contact regular $r$-cubic configurations on $\left(J^{1}\left(\mathbb{R} \times \mathbb{R}^{n}, \mathbb{R}\right), w_{i}\right)\left(\Pi\left(w_{i}\right)=0\right)$ and $\left\{L_{\sigma, t}^{i}\right\}_{\sigma \subset I_{r}, t \in(\mathbb{R}, 0)}$ be the corresponding oneparameter families of contact regular $r$-cubic configurations on $J^{1}\left(\mathbb{R}^{n}, \mathbb{R}\right)$ respectively. We take the smooth path germs $w_{i}:(\mathbb{R}, 0) \rightarrow\left(J^{1}\left(\mathbb{R}^{n}, \mathbb{R}\right), 0\right)$ such that $\left\{L_{\sigma, t}^{i}\right\}_{\sigma \subset I_{r}}$ are defined at $w_{i}(t)$ for $i=1,2$. Suppose that there exists a $\mathcal{P}$-Legendrian equivalence $K$ from $\left\{\tilde{L}_{\sigma}^{1}\right\}_{\sigma \subset I_{r}}$ to $\left\{\tilde{L}_{\sigma}^{2}\right\}_{\sigma \subset I_{r}}$ of the form (4). We set $W_{\sigma, t}^{i}$ be the wavefront of $L_{\sigma, t}^{i}$ for $\sigma \subset I_{r}, t \in(\mathbb{R}, 0)$ and $i=1,2$. We define the family of diffeomorphism $g_{t}:\left(\mathbb{R}^{n} \times \mathbb{R}, \pi\left(w_{1}(t)\right)\right) \rightarrow\left(\mathbb{R}^{n} \times \mathbb{R}, \pi\left(w_{2}(t)\right)\right)$ by $g_{t}(q, z)=\left(\phi_{2}(t, q, z), \phi_{3}(t, q, z)\right)$. Then we have that $g_{t}\left(W_{\sigma, t}^{1}\right)=W_{\sigma, \phi_{1}(t)}^{1}$ for all $\sigma \subset I_{r}, t \in(\mathbb{R}, 0)$.

We also define the equivalence relation among reticular Legendrian unfoldings. Let $\mathcal{L}_{i}:\left(\mathbb{L}^{r}, 0\right) \rightarrow\left(J^{1}\left(\mathbb{R} \times \mathbb{R}^{n}, \mathbb{R}\right), w_{i}\right),(i=1,2)$ be reticular Legendrian unfoldings. We say that $\mathcal{L}_{1}$ and $\mathcal{L}_{2}$ are $\mathcal{P}$-Legendrian equivalent if there exist a $\mathcal{P}$-Legendrian equivalence $K$ and a reticular $r$-diffeomorphism $\Psi$ such that $K \circ \mathcal{L}_{1}=\mathcal{L}_{2} \circ \Psi$.

We remark that two reticular Legendrian unfoldings are $\mathcal{P}$-Legendrian equivalent if and only if the corresponding unfolded contact regular $r$-cubic configurations are $\mathcal{P}$-Legendrian equivalent.

By the same proof of Lemma 5.3 in [6], we have the following:

LEMMA 3.4. Let $\left\{\tilde{L}_{\sigma}\right\}_{\sigma \subset I_{r}}$ be an unfolded contact regular $r$-cubic configuration on $\left(J^{1}\left(\mathbb{R} \times \mathbb{R}^{n}, \mathbb{R}\right), w\right)(\Pi(w)=0)$. Then there exists a $\mathcal{P}$-contact diffeomorphism germ $C$ on $\left(J^{1}\left(\mathbb{R} \times \mathbb{R}^{n}, \mathbb{R}\right), 0\right)$ such that $C$ defines $\left\{\tilde{L}_{\sigma}\right\}_{\sigma \subset I_{r}}$ and preserves the canonical 1-form. 
By this lemma we may assume that all reticular Legendrian unfoldings (and all unfolded contact regular $r$-cubic configurations) are defined by $\mathcal{P}$-contact diffeomorphism germs which preserve the canonical 1-form.

We can construct generating families of reticular Legendrian unfoldings. A function germ $F(x, y, t, q, z) \in \mathfrak{M}(r ; k+1+n+1)$ is said to be $\mathcal{P}$-C-non-degenerate if $\frac{\partial F}{\partial x}(0)=\frac{\partial F}{\partial y}(0)=0$ and $x, t, F, \frac{\partial F}{\partial x}, \frac{\partial F}{\partial y}$ are independent on $\left(\mathbb{H}^{k} \times \mathbb{R}^{k+1+n+1}, 0\right)$.

A $\mathcal{P}$-C -non-degenerate function germ $F(x, y, t, q, z) \in \mathfrak{M}(r ; k+1+n+1)$ is called a generating family of a reticular Legendrian unfoldings $\mathcal{L}$ if

$$
\begin{aligned}
\mathcal{L}\left(\tilde{L}_{\sigma}^{r, 0}\right)=\left\{\left(t, q, z, \frac{\partial F}{\partial t} /\left(-\frac{\partial F}{\partial z}\right), \frac{\partial F}{\partial q} /\left(-\frac{\partial F}{\partial z}\right)\right) \in\left(J^{1}\left(\mathbb{R} \times \mathbb{R}^{n}, \mathbb{R}\right), w\right) \mid\right. \\
\left.x_{\sigma}=F=\frac{\partial F}{\partial x_{I_{r}-\sigma}}=\frac{\partial F}{\partial y}=0, x_{I_{r}-\sigma} \geq 0\right\} \text { for all } \sigma \subset I_{r} .
\end{aligned}
$$

We remark that for a $\mathcal{P}$ - $C$-non-degenerate function germ $F(x, y, t, q, z)$, the function germ $F(\cdot, \cdot, t, \cdot, \cdot)$ is $C$-non-degenerate (see $[6, \mathrm{p} .111]$ ).

LEMmA 3.5. Let $C$ be a $\mathcal{P}$-contact diffeomorphism germ $\left(J^{1}\left(\mathbb{R} \times \mathbb{R}^{n}, \mathbb{R}\right), 0\right) \rightarrow$ $\left(J^{1}\left(\mathbb{R} \times \mathbb{R}^{n}, \mathbb{R}\right), w\right)(\Pi(w)=0)$ which preserves the canonical 1 -form. If the map germ

$$
(T, Q, Z, S, P) \rightarrow\left(T, Q, Z, s_{C}(T, Q, Z, S, P), p_{C}(T, Q, Z, S, P)\right)
$$

is diffeomorphism, then there exists a function germ $H(T, Q, p) \in \mathfrak{M}(1+n+n)^{2}$ such that the canonical relation $P_{C}$ associated with $C$ has the form:

$$
\begin{aligned}
& P_{C}=\{(T, Q, Z,- \frac{\partial H}{\partial T}(T, Q, p)+s,-\frac{\partial H}{\partial Q}, \\
&\left.\left.T,-\frac{\partial H}{\partial p}, H-\left\langle\frac{\partial H}{\partial p}, p+p^{0}\right\rangle+s^{0} T+Z, s+s^{0}, p+p^{0}\right)\right\},
\end{aligned}
$$

and the function germ $F \in \mathfrak{M}(r ; n+1+n+1)$ defined by $F(x, y, t, q, z)=-z+$ $H(t, x, 0, y)+\left\langle y+p^{0}, q\right\rangle+s^{0} t$ is a generating family of the reticular Legendrian unfolding $\left.C\right|_{\mathbb{L}}$.

Proof. We have that $d z-\left(s+s^{0}\right) d t-\left(p+p^{0}\right) d q=d Z-S d T-P d Q$ on $P_{C}$. It follows that $d(z-Z)=\left(s+s^{0}\right) d t+\left(p+p^{0}\right) d q-S d T-P d Q$ and $d(z-Z-(s+$ $\left.\left.s^{0}\right) t-\left(p+p^{0}\right) q\right)=-t d s-q d p-S d T-P d Q$. Then there exists a function germ $H^{\prime}(T, Q, s, p) \in \mathfrak{M}(1+n+1+1+n)^{2}$ such that

$z-Z-\left(s+s^{0}\right) t-\left(p+p^{0}\right) q=H^{\prime}(T, Q, s, p), t=-\frac{\partial H^{\prime}}{\partial s}, q=-\frac{\partial H^{\prime}}{\partial p}, S=-\frac{\partial H^{\prime}}{\partial T}, P=-\frac{\partial H^{\prime}}{\partial Q}$

on $P_{C}$. Since $t=T=-\frac{\partial H^{\prime}}{\partial s}$ on $P_{C}$, we have that $H^{\prime}(T, Q, s, p)=H(T, Q, p)-T s$ for some $H(T, Q, p) \in \mathfrak{M}(1+n+n)^{2}$. Then we have that

$$
z-Z-T\left(s+s^{0}\right)-\left\langle-\frac{\partial H}{\partial p}, p+p^{0}\right\rangle=H(T, Q, p)-T s .
$$

It follows that

$$
z=H(T, Q, p)-\left\langle\frac{\partial H}{\partial p}, p+p^{0}\right\rangle+s^{0} T+Z .
$$


Then we have the required form of $P_{C}$. By the direct calculation with the form $P_{C}$, we have that $F$ is a generating family of $\left.C\right|_{\mathbb{L}^{r}}$.

We have the following theorem which gives the relations between reticular Legendrian unfoldings and their generating families.

TheOREM 3.6. (1) For any reticular Legendrian unfolding $\mathcal{L}:\left(\mathbb{L}^{r}, 0\right) \rightarrow\left(J^{1}(\mathbb{R} \times\right.$ $\left.\left.\mathbb{R}^{n}, \mathbb{R}\right), w\right)(\Pi(w)=0)$, there exists a function germ $F(x, y, t, q, z) \in \mathfrak{M}(r ; k+1+n+1)$ which is a generating family of $\mathcal{L}$.

(2) For any $\mathcal{P}-C$-non-degenerate function $\operatorname{germ} F(x, y, t, q, z) \in \mathfrak{M}(r ; k+1+n+1)$ with $\frac{\partial F}{\partial t}(0)=\frac{\partial F}{\partial q}(0)=0$, there exists a reticular Legendrian unfolding $\mathcal{L}:\left(\mathbb{L}^{r}, 0\right) \rightarrow$ $\left(J^{1}\left(\mathbb{R} \times \mathbb{R}^{n}, \mathbb{R}\right), w\right)(\Pi(w)=0)$ of which $F$ is a generating family.

(3) Two reticular Legendrian unfolding are $\mathcal{P}$-Legendrian equivalent if and only if their generating families are stably reticular $t-\mathcal{P}-\mathcal{K}$-equivalent.

This theorem is proved by analogous methods of [5], [6]. We give the sketch of the proof. We may assume that $w=0$.

(1) Let $C$ be a $\mathcal{P}$-contact diffeomorphism germ on $\left(J^{1}\left(\mathbb{R} \times \mathbb{R}^{n}, \mathbb{R}\right), 0\right)$ such that $\left.C\right|_{\mathbb{L}}=$ $\mathcal{L}$. We may assume that $C^{*} \Theta=\Theta$. By taking a $\mathcal{P}$-Legendrian equivalence of $\mathcal{L}$, we may assume that the canonical relation $P_{C}$ associated with $C$ has the form (5) for the function germ $H \in \mathfrak{M}(1+n+n)^{2}$. Then the function germ $F(x, y, t, q, z) \in$ $\mathfrak{M}(r ; n+1+n+1)$ defined by

$$
F(x, y, t, q, z)=-z+H\left(t, x_{1}, \ldots, x_{r}, 0, y\right)+\langle y, q\rangle
$$

is a generating family of $\mathcal{L}$.

(2) Let a $\mathcal{P}$ - $C$-non-degenerate function germ $F(x, y, t, q, z) \in \mathfrak{M}(r ; k+1+n+1)$ with $\frac{\partial F}{\partial t}(0)=\frac{\partial F}{\partial q}(0)=0$ be given. By [6, Lemma 2.1], we may assume that $F$ has the form $F(x, y, t, q, z)=-z+F_{0}(x, y, t, q)$ for some $F_{0} \in \mathfrak{M}(r ; k+1+n)$. Choose an $(n-r) \times k$-matrix $A$ and an $(n-r) \times n$-matrix $B$ such that the matrix

$$
\left(\begin{array}{ccc}
\frac{\partial^{2} F_{0}}{\partial x \partial y} & \frac{\partial^{2} F_{0}}{\partial x \partial q} & \frac{\partial^{2} F_{0}}{\partial x \partial t} \\
\frac{\partial^{2} F_{0}}{\partial y \partial y} & \frac{\partial^{2} F_{0}}{\partial y \partial q} & \frac{\partial^{2} F_{0}}{\partial y \partial t} \\
A & B & 0 \\
0 & 0 & 1
\end{array}\right)_{0} \text { is invertible. }
$$

Let $F^{\prime} \in \mathfrak{M}(r+k+1+n+1)$ be a function germ which is obtained by an extension the source space of $F$ to $\left(\mathbb{R}^{r+k+1+n+1}, 0\right)$. Define the function $G(S, Q, y, t, q, z) \in$ $\mathfrak{M}(n+1+1+k+1+n+1)$ by that

$$
\begin{aligned}
& G(Q, Z, S, y, t, q, z)=-z+F^{\prime}\left(Q_{1}, \ldots, Q_{r}, y, t, q\right)+ \\
& \left(Q_{r+1}, \ldots, Q_{n}\right) A\left(\begin{array}{c}
y_{1} \\
\vdots \\
y_{k}
\end{array}\right)+\left(Q_{r+1}, \ldots, Q_{n}\right) B\left(\begin{array}{c}
q_{1} \\
\vdots \\
q_{n}
\end{array}\right)+S t .
\end{aligned}
$$

Then $G$ is a generating family of the canonical relation $P_{C}$ associated with some $\mathcal{P}$ contact diffeomorphism germ $C$. The function germ $F$ is a generating family of the reticular Legendrian unfolding $\left.C\right|_{\mathbb{L}}$. 
(3) We need only to prove that: If $F_{1}, F_{2} \in \mathfrak{M}(r ; k+1+n)$ are generating families of the same reticular Legendrian unfolding, then they are reticular $t-\mathcal{P}-\mathcal{K}$-equivalent. We may reduce that $F_{i}$ has the form $F_{i}(x, y, t, q, z)=-z+F_{i}^{0}(x, y, t, q)$ for $F_{i}^{0} \in$ $\mathfrak{M}(r ; k+1+n), i=1,2$. Then $F_{1}^{0}$ and $F_{2}^{0}$ are generating families of the same reticular Lagrangian map in the sense of [5]. By [5, p.587 the assertion (3)], there exists a reticular $\mathcal{R}$-equivalence from $F_{2}^{0}$ to $F_{1}^{0}$ of the form:

$$
F_{1}^{0}(x, y, t, q)=F_{2}^{0}\left(x \phi_{1}(x, y, t, q), \phi_{2}(x, y, t, q), t, q\right) .
$$

This means that $F_{1}$ and $F_{2}$ are reticular $t-\mathcal{P}$ - $\mathcal{K}$-equivalent.

4. Multi-reticular Legendrian unfoldings. Let $\mathcal{L}_{i}:\left(\mathbb{L}^{r_{i}}, 0\right) \rightarrow\left(J^{1}(\mathbb{R} \times\right.$ $\left.\left.\mathbb{R}^{n}, \mathbb{R}\right), w_{i}\right)(i=1, \ldots, m)$ be reticular Legendrian unfoldings with $\Pi\left(w_{i}\right)=0$ where $w_{1}, \ldots, w_{m}$ are distinct. Then we call $\mathcal{L}=\left(\mathcal{L}_{1}, \ldots, \mathcal{L}_{m}\right)$ a multi-reticular Legendrian unfolding.

Let $\left(\mathcal{L}_{1}, \ldots, \mathcal{L}_{m}\right)$ and $\left(\mathcal{L}_{1}^{\prime}, \ldots, \mathcal{L}_{m}^{\prime}\right)$ be multi-reticular Legendrian unfoldings. We say that they are $\mathcal{P}_{(m)}$-Legendrian equivalent if there exist contact diffeomorphism germs

$$
K_{i}:\left(J^{1}\left(\mathbb{R} \times \mathbb{R}^{n}, \mathbb{R}\right), w_{i}\right) \rightarrow\left(J^{1}\left(\mathbb{R} \times \mathbb{R}^{n}, \mathbb{R}\right), w_{i}^{\prime}\right) \quad(i=1, \ldots, m)
$$

of the forms

$$
K_{i}(t, q, z, s, p)=\left(\phi_{1}(t), \phi_{2}(t, q, z), \phi_{3}(t, q, z), \phi_{4}^{i}(t, q, z, s, p), \phi_{5}^{i}(t, q, z, s, p)\right)
$$

and reticular $r_{i}$-diffeomorphisms $\Psi_{i}$ on $\left(\mathbb{L}^{r_{i}}, 0\right)$ such that $K_{i} \circ \mathcal{L}_{i}=\mathcal{L}_{i}^{\prime} \circ \Psi_{i}$ for $i=$ $1, \ldots, m$.

Let $\left(\mathcal{L}_{1}, \ldots, \mathcal{L}_{m}\right)$ be a multi-reticular Legendrian unfolding and $F_{i} \in \mathfrak{M}\left(r_{i} ; k_{i}+\right.$ $1+n+1)$ be generating families of $\mathcal{L}_{i}$ for $i=1, \ldots, m$. We call $F=\left(F_{1}, \ldots, F_{m}\right) a$ generating family of $\left(\mathcal{L}_{1}, \ldots, \mathcal{L}_{m}\right)$.

By the consideration of stable reticular $t-(\mathcal{P}-\mathcal{K})_{(m)}$-equivalences of generating families of multi-reticular Legendrian unfoldings, we may assume that $r=r_{1}=\cdots=r_{m}, \quad k=k_{1}=\cdots=k_{m}$ by taking stable reticular $t-(\mathcal{P}-\mathcal{K})_{(m)^{-}}$ equivalences of them.

We say that a map germ $\left(F_{1}, \ldots, F_{m}\right) \in \mathfrak{M}(r ; k+1+n+$ $1, m)$ is $\mathcal{P}_{(m)}-C$-non-degenerate if all $F_{i}$ are $\mathcal{P}$-C-non-degenerate and $\left(\frac{\partial F_{1}}{\partial t} /\left(-\frac{\partial F_{1}}{\partial z}\right), \frac{\partial F_{1}}{\partial q} /\left(-\frac{\partial F_{1}}{\partial z}\right)\right), \ldots,\left(\frac{\partial F_{m}}{\partial t} /\left(-\frac{\partial F_{m}}{\partial z}\right), \frac{\partial F_{m}}{\partial q} /\left(-\frac{\partial F_{m}}{\partial z}\right)\right)$ are distinct.

By Theorem 3.6, we have the following:

TheOREM 4.1. (1) For any multi-reticular Legendrian unfolding $\mathcal{L}$, there exists a multi-generating family of $\mathcal{L}$,

(2) For any $\mathcal{P}_{(m)}$-C-non-degenerate map germ $F=\left(F_{1}, \ldots, F_{m}\right) \in \mathfrak{M}(r ; k+1+$ $n+1, m)$, there exists a multi-reticular Legendrian unfolding of which $F$ is a multigenerating family,

(3) Let $F=\left(F_{1}, \ldots, F_{m}\right)$ and $F^{\prime}=\left(F_{1}^{\prime}, \ldots, F_{m}^{\prime}\right)$ be multi-generating families of multireticular Legendrian unfoldings $\left(\mathcal{L}_{1}, \ldots, \mathcal{L}_{m}\right)$ and $\left(\mathcal{L}_{1}^{\prime}, \ldots, \mathcal{L}_{m}^{\prime}\right)$ respectively. Then $\left(\mathcal{L}_{1}, \ldots, \mathcal{L}_{m}\right)$ and $\left(\mathcal{L}_{1}^{\prime}, \ldots, \mathcal{L}_{m}^{\prime}\right)$ are $\mathcal{P}_{(m)}$-Legendrian equivalent if and only if $F$ and $F^{\prime}$ are stably reticular $t-(\mathcal{P}-\mathcal{K})_{(m)}$-equivalent.

Let $U$ be an open set in $J^{1}\left(\mathbb{R} \times \mathbb{R}^{n}, \mathbb{R}\right)$. We consider contact embedding germs $\left(J^{1}\left(\mathbb{R} \times \mathbb{R}^{n}, \mathbb{R}\right), 0\right) \rightarrow J^{1}\left(\mathbb{R} \times \mathbb{R}^{n}, \mathbb{R}\right)$ and contact embeddings $U \rightarrow J^{1}\left(\mathbb{R} \times \mathbb{R}^{n}, \mathbb{R}\right)$. Let 
$(T, Q, S, Z, P)$ and $(t, q, z, s, p)$ be canonical coordinates of the source space and the target space respectively. We define the following notations:

$\imath:\left(J^{1}\left(\mathbb{R} \times \mathbb{R}^{n}, \mathbb{R}\right) \cap\{Z=0\}, 0\right) \rightarrow\left(J^{1}\left(\mathbb{R} \times \mathbb{R}^{n}, \mathbb{R}\right), 0\right)$ be the inclusion map on the source space,

$$
\begin{aligned}
& C_{T}\left(J^{1}\left(\mathbb{R} \times \mathbb{R}^{n}, \mathbb{R}\right), 0\right)=\{C \mid C \text { is a } \mathcal{P} \text {-contact embedding germ } \\
& \left.\left(J^{1}\left(\mathbb{R} \times \mathbb{R}^{n}, \mathbb{R}\right), 0\right) \rightarrow J^{1}\left(\mathbb{R} \times \mathbb{R}^{n}, \mathbb{R}\right)\right\}, \\
& C_{T}^{\Theta}\left(J^{1}\left(\mathbb{R} \times \mathbb{R}^{n}, \mathbb{R}\right), 0\right)=\left\{C \in C_{T}\left(J^{1}\left(\mathbb{R} \times \mathbb{R}^{n}, \mathbb{R}\right), 0\right) \mid C^{*} \Theta=\Theta\right\}, \\
& C_{T}^{Z}\left(J^{1}\left(\mathbb{R} \times \mathbb{R}^{n}, \mathbb{R}\right), 0\right)=\left\{C \circ \imath \mid C \in C_{T}\left(J^{1}\left(\mathbb{R} \times \mathbb{R}^{n}, \mathbb{R}\right), 0\right)\right\}, \\
& C_{T}^{\Theta, Z}\left(J^{1}\left(\mathbb{R} \times \mathbb{R}^{n}, \mathbb{R}\right), 0\right)=\left\{C \circ \imath \mid C \in C_{T}^{\Theta}\left(J^{1}\left(\mathbb{R} \times \mathbb{R}^{n}, \mathbb{R}\right), 0\right)\right\} .
\end{aligned}
$$

Let $V=U \cap\{Z=0\}$ and $\tilde{\imath}: V \rightarrow U$ be the inclusion map.

$$
\begin{aligned}
& C_{T}\left(U, J^{1}\left(\mathbb{R} \times \mathbb{R}^{n}, \mathbb{R}\right)\right)=\left\{\tilde{C}: U \rightarrow J^{1}\left(\mathbb{R} \times \mathbb{R}^{n}, \mathbb{R}\right) \mid\right. \\
&\tilde{C} \text { is a contact embedding of the form (3) }\}, \\
& C_{T}^{\Theta}\left(U, J^{1}\left(\mathbb{R} \times \mathbb{R}^{n}, \mathbb{R}\right)\right)=\left\{\tilde{C} \in C_{T}\left(U, J^{1}\left(\mathbb{R} \times \mathbb{R}^{n}, \mathbb{R}\right)\right) \mid \tilde{C}^{*} \Theta=\Theta\right\}, \\
& C_{T}^{Z}\left(V, J^{1}\left(\mathbb{R} \times \mathbb{R}^{n}, \mathbb{R}\right)\right)=\left\{\tilde{C} \circ \tilde{\imath} \mid \tilde{C} \in C_{T}\left(U, J^{1}\left(\mathbb{R} \times \mathbb{R}^{n}, \mathbb{R}\right)\right)\right\}, \\
& C_{T}^{\Theta, Z}\left(V, J^{1}\left(\mathbb{R} \times \mathbb{R}^{n}, \mathbb{R}\right)\right)=\left\{\tilde{C} \circ \tilde{\imath} \mid \tilde{C} \in C_{T}^{\Theta}\left(U, J^{1}\left(\mathbb{R} \times \mathbb{R}^{n}, \mathbb{R}\right)\right)\right\} .
\end{aligned}
$$

Definition 4.2. We define stabilities of multi-reticular Legendrian unfoldings. Let $\mathcal{L}=\left(\mathcal{L}_{1}, \ldots, \mathcal{L}_{m}\right)$ be a multi-reticular Legendrian unfolding.

Stability. We say that $\mathcal{L}$ is stable if the following condition holds: Let $C^{0, i} \in$ $C_{T}\left(J^{1}\left(\mathbb{R} \times \mathbb{R}^{n}, \mathbb{R}\right), 0\right)$ be $\mathcal{P}$-contact embedding germs such that $\left.C^{0, i}\right|_{\mathbb{L}^{r_{i}}}=\mathcal{L}_{i}$ and $\tilde{C}^{0, i} \in C_{T}\left(U_{i}, J^{1}\left(\mathbb{R} \times \mathbb{R}^{n}, \mathbb{R}\right)\right)$ be representatives of $C^{0, i}$ for $i=1, \ldots, m$. Then there exist open neighborhoods $N_{\tilde{C}^{0, i}}$ of $\tilde{C}^{0, i}$ in $C^{\infty}$-topology for $i=1, \ldots, m$ such that for any $\tilde{C}^{i} \in N_{\tilde{C}^{0, i}}$, there exist points $x_{i}=\left(T^{i}, 0, \ldots, 0, P_{r+1}^{i}, \ldots, P_{n}^{i}\right) \in U_{i}$ such that the multi-reticular Legendrian unfolding $\left(\mathcal{L}_{x_{1}}^{1}, \ldots, \mathcal{L}_{x_{m}}^{m}\right)$ and $\mathcal{L}$ are $\mathcal{P}_{(m)}$-Legendrian equivalent, where the reticular Legendrian unfolding $\mathcal{L}_{x_{i}}^{i}$ is defined by

$$
x=(T, Q, Z, S, P) \mapsto \tilde{C}^{i}\left(x_{0}+x\right)-\tilde{C}^{i}\left(x_{0}\right)+\left(0,0, P_{r+1}^{i} Q_{r+1}+\cdots+P_{n}^{i} Q_{n}, 0,0\right) .
$$

Homotopical stability. A one-parameter family of $\mathcal{P}$-contact embedding germs $\bar{C}:\left(J^{1}\left(\mathbb{R} \times \mathbb{R}^{n}, \mathbb{R}\right) \times \mathbb{R},(0,0)\right) \rightarrow J^{1}\left(\mathbb{R} \times \mathbb{R}^{n}, \mathbb{R}\right)\left((T, Q, Z, S, P, \tau) \mapsto C_{\tau}(T, Q, Z, S, P)\right)$ is called a $\mathcal{P}$-contact deformation of $\mathcal{L}$ if $\left.C_{0}\right|_{\mathbb{L}}=\mathcal{L}$. A map germ $\bar{\Psi}:\left(J^{1}\left(\mathbb{R} \times \mathbb{R}^{n}, \mathbb{R}\right) \times\right.$ $\mathbb{R},(0,0)) \rightarrow\left(J^{1}\left(\mathbb{R} \times \mathbb{R}^{n}, \mathbb{R}\right), 0\right)\left((T, Q, Z, S, P, \tau) \mapsto \Psi_{\tau}(T, Q, Z, S, P)\right)$ is called a oneparameter deformation of reticular r-diffeomorphisms if $\Psi_{0}=i d_{J^{1}\left(\mathbb{R} \times \mathbb{R}^{n}, \mathbb{R}\right)}$ and $\Psi_{t}$ is a reticular $r$-diffeomorphism for all $t$ around 0 . We say that $\mathcal{L}$ is homotopically stable if for any reticular $\mathcal{P}$-contact deformations $\bar{C}^{i}=\left\{C_{\tau}^{i}\right\}$ of $\mathcal{L}_{i}$, there exist oneparameter families of $\mathcal{P}$-Legendrian equivalences $\bar{K}^{i}=\left\{K_{\tau}^{i}\right\}$ on $\left(J^{1}\left(\mathbb{R} \times \mathbb{R}^{n}, \mathbb{R}\right), w_{i}\right)$ with $K_{0}^{i}=i d$ of the form

$$
K_{\tau}^{i}(t, q, z, s, p)=\left(\phi_{\tau}^{1}(t), \phi_{\tau}^{2}(t, q, z), \phi_{\tau}^{3}(t, q, z), \phi_{\tau}^{4, i}(t, q, z, s, p), \phi_{\tau}^{5, i}(t, q, z, s, p)\right)
$$

and one-parameter deformations of reticular $r_{i}$-diffeomorphisms $\bar{\Psi}^{i}=\left\{\Psi_{\tau}^{i}\right\}$ such that $C_{\tau}^{i}=K_{\tau}^{i} \circ C_{0}^{i} \circ \Psi_{\tau}^{i}$ for $t$ around 0 and $i=1, \ldots, m$. 
Infinitesimal stability. Let $C \in C_{T}\left(J^{1}\left(\mathbb{R} \times \mathbb{R}^{n}, \mathbb{R}\right), 0\right)$ be a $\mathcal{P}$-contact diffeomorphism germ. We say that a vector field $v$ on $\left(J^{1}\left(\mathbb{R} \times \mathbb{R}^{n}, \mathbb{R}\right), w\right)(\Pi(w)=0)$ along $C$ is an infinitesimal $\mathcal{P}$-contact transformation of $C$ if there exists a $\mathcal{P}$-contact deformation $\bar{C}=\left\{C_{\tau}\right\}$ on $\left(J^{1}\left(\mathbb{R} \times \mathbb{R}^{n}, \mathbb{R}\right), 0\right)$ such that $C_{0}=C$ and $\left.\frac{d C_{\tau}}{d \tau}\right|_{\tau=0}=v$. We say that a vector field $\xi$ on $\left(J^{1}\left(\mathbb{R} \times \mathbb{R}^{n}, \mathbb{R}\right), 0\right)$ is an infinitesimal reticular $r$-diffeomorphism if there exists a one-parameter deformation of reticular $r$-diffeomorphisms $\bar{\Psi}=\left\{\Psi_{\tau}\right\}$ such that $\left.\frac{d \Psi_{\tau}}{d \tau}\right|_{\tau=0}=\xi$. We say that a vector field $\eta$ on $\left(J^{1}\left(\mathbb{R} \times \mathbb{R}^{n}, \mathbb{R}\right), w\right)$ is an infinitesimal $\mathcal{P}$-Legendrian equivalence if there exists a one-parameter family of $\mathcal{P}$-Legendrian equivalences $\bar{K}=\left\{K_{\tau}\right\}$ such that $K_{0}=i d_{J^{1}\left(\mathbb{R} \times \mathbb{R}^{n}, \mathbb{R}\right)}$ and $\left.\frac{d K_{\tau}}{d \tau}\right|_{\tau=0}=\eta$. We say that $\mathcal{L}$ is infinitesimally stable if for any extension $C^{i}$ of $\mathcal{L}_{i}$ and any infinitesimal $\mathcal{P}$-contact transformation $v_{i}$ of $C^{i}$, there exist infinitesimal reticular $r_{i}$-diffeomorphisms $\xi_{i}$ and infinitesimal $\mathcal{P}$-Legendrian equivalences $\eta_{i}$ at $w_{i}$ of the form

$$
\begin{aligned}
\eta_{i}(t, q, z, s, p)=a_{1}(t) \frac{\partial}{\partial t}+a_{2}( & t, q, z) \frac{\partial}{\partial q}+a_{3}(t, q, z) \frac{\partial}{\partial z} \\
& +a_{4}^{i}(t, q, z, s, p) \frac{\partial}{\partial s}+a_{5}^{i}(t, q, z, s, p) \frac{\partial}{\partial p}
\end{aligned}
$$

such that $v_{i}=C_{*}^{i} \xi_{i}+\eta_{i} \circ C^{i}$ for $i=1, \ldots, m$.

We denote $C_{T}\left(U, J^{1}\left(\mathbb{R} \times \mathbb{R}^{n}, \mathbb{R}\right)\right)^{(m)}=C_{T}\left(U, J^{1}\left(\mathbb{R} \times \mathbb{R}^{n}, \mathbb{R}\right)\right) \times \cdots \times C_{T}\left(U, J^{1}(\mathbb{R} \times\right.$ $\left.\mathbb{R}^{n}, \mathbb{R}\right)$ ) (m-products) and denote other notations analogously. We call an element $C=\left(C_{1}, \ldots, C_{m}\right) \in C_{T}\left(J^{1}\left(\mathbb{R} \times \mathbb{R}^{n}, \mathbb{R}\right), 0\right)^{(m)}$ a $\mathcal{P}_{(m)}$-contact diffeomorphism germ if $\Pi \circ C(0)=0$ and $C_{1}(0), \ldots, C_{m}(0)$ are distinct.

We may take an extension of a reticular Legendrian unfolding $\mathcal{L}$ by an element of $C_{T}^{\Theta}\left(J^{1}\left(\mathbb{R} \times \mathbb{R}^{n}, \mathbb{R}\right), 0\right)$ by Lemma 3.4. Then as the remark after the definition of the stability of reticular Legendrian maps in [6, p.121], we may consider the following other definitions of stabilities of multi-reticular Legendrian unfoldings: (1) The definition given by replacing $C_{T}\left(J^{1}\left(\mathbb{R} \times \mathbb{R}^{n}, \mathbb{R}\right), 0\right)$ and $C_{T}\left(U_{i}, J^{1}\left(\mathbb{R} \times \mathbb{R}^{n}, \mathbb{R}\right)\right)$ to $C_{T}^{\Theta}\left(J^{1}\left(\mathbb{R} \times \mathbb{R}^{n}, \mathbb{R}\right), 0\right)$ and $C_{T}^{\Theta}\left(U_{i}, J^{1}\left(\mathbb{R} \times \mathbb{R}^{n}, \mathbb{R}\right)\right)$ of original definition respectively. $(2)$ The definition given by replacing to $C_{T}^{Z}\left(J^{1}\left(\mathbb{R} \times \mathbb{R}^{n}, \mathbb{R}\right), 0\right)$ and $C_{T}^{Z}\left(V_{i}, J^{1}\left(\mathbb{R} \times \mathbb{R}^{n}, \mathbb{R}\right)\right)$ respectively. (3) The definition given by replacing to $C_{T}^{\Theta, Z}\left(J^{1}\left(\mathbb{R} \times \mathbb{R}^{n}, \mathbb{R}\right), 0\right)$ and $C_{T}^{\Theta, Z}\left(V_{i}, J^{1}\left(\mathbb{R} \times \mathbb{R}^{n}, \mathbb{R}\right)\right)$ respectively, where $V_{i}=U_{i} \cap\{Z=0\}$.

Then we have the following lemma which is proved by the same method of the proof of [6, Lemma 7.2]

LEMmA 4.3. The original definition and other three definitions of stabilities of multi-reticular Legendrian unfoldings are all equivalent.

By this lemma, we may choose an extension of a multi-reticular Legendrian unfolding from among all of $\left.\left.C_{T}\left(J^{1}\left(\mathbb{R} \times \mathbb{R}^{n}, \mathbb{R}\right), 0\right)\right)^{(m)}, C_{T}^{\Theta}\left(J^{1}\left(\mathbb{R} \times \mathbb{R}^{n}, \mathbb{R}\right), 0\right)\right)^{(m)}$, $\left.C_{T}^{Z}\left(J^{1}\left(\mathbb{R} \times \mathbb{R}^{n}, \mathbb{R}\right), 0\right)\right)^{(m)}$, and $\left.C_{T}^{\Theta, Z}\left(J^{1}\left(\mathbb{R} \times \mathbb{R}^{n}, \mathbb{R}\right), 0\right)\right)^{(m)}$.

We say that a function germ $H$ on $\left(J^{1}\left(\mathbb{R} \times \mathbb{R}^{n}, \mathbb{R}\right), 0\right)$ is $\mathcal{P}$-fiber preserving if $H$ has the form $H(t, q, z, s, p)=\sum_{j=1}^{n} h_{j}(t, q, z) p_{j}+h_{0}(t, q, z)+a(t) s$.

LEMma 4.4. Let $C \in C_{T}\left(J^{1}\left(\mathbb{R} \times \mathbb{R}^{n}, \mathbb{R}\right), 0\right)$. Then the following hold: (1) A vector field germ $v$ on $\left(J^{1}\left(\mathbb{R} \times \mathbb{R}^{n}, \mathbb{R}\right), 0\right)$ along $C$ is an infinitesimal $\mathcal{P}$-contact transformation of $C$ if and only if there exists a function germ $f$ on $\left(J^{1}\left(\mathbb{R} \times \mathbb{R}^{n}, \mathbb{R}\right), 0\right)$ such that $f$ 
does not depend on $s$ and $v=X_{f} \circ C$.

(2) A vector field germ $\eta$ on $\left(J^{1}\left(\mathbb{R} \times \mathbb{R}^{n}, \mathbb{R}\right), 0\right)$ is an infinitesimal $\mathcal{P}$-Legendrian equivalence if and only if there exists a $\mathcal{P}$-fiber preserving function germ $H$ on $\left(J^{1}(\mathbb{R} \times\right.$ $\left.\left.\mathbb{R}^{n}, \mathbb{R}\right), 0\right)$ such that $\eta=X_{H}$.

(3) A vector field $\xi$ on $\left(J^{1}\left(\mathbb{R} \times \mathbb{R}^{n}, \mathbb{R}\right), 0\right)$ is an infinitesimal reticular $r$-diffeomorphism if and only if there exists a function germ $g_{i} \in B^{r}$ such that $\xi=X_{g}$, where $B^{r}=$ $\left\langle q_{1} p_{1}, \ldots, q_{r} p_{r}, q_{r+1}, \ldots, q_{n}, z\right\rangle_{\mathcal{E}_{t, q, z, p}}+\langle s\rangle_{\mathcal{E}_{t}}$.

Let $U$ be a neighborhood of 0 in $J^{1}\left(\mathbb{R} \times \mathbb{R}^{n}, \mathbb{R}\right)$. We define:

$J_{C_{T}^{\Theta}}^{l}\left(U, J^{1}\left(\mathbb{R} \times \mathbb{R}^{n}, \mathbb{R}\right)\right)=\left\{j^{l} C\left(w_{0}\right) \in J^{l}\left(U, J^{1}\left(\mathbb{R} \times \mathbb{R}^{n}, \mathbb{R}\right)\right) \mid\right.$

$C:\left(U, w_{0}\right) \rightarrow J^{1}\left(\mathbb{R} \times \mathbb{R}^{n}, \mathbb{R}\right)$ is a $\mathcal{P}$-contact embedding germ which preserves $\left.\Theta\right\}$.

TheOREM 4.5. Let $Q_{i}, i=1,2, \ldots$ are submanifolds of $J_{C_{T}^{\Theta}}^{l}\left(U, J^{1}\left(\mathbb{R} \times \mathbb{R}^{n}, \mathbb{R}\right)\right)^{(m)}$. Then the set

$T=\left\{C=\left(C_{1}, \ldots, C_{m}\right) \in C_{T}^{\Theta}\left(U, J^{1}\left(\mathbb{R} \times \mathbb{R}^{n}, \mathbb{R}\right)\right)^{(m)} \mid j^{l} C\right.$ is transversal to $Q_{i}$ for all $\left.i \in \mathbb{N}\right\}$

is a residual set in $C_{T}^{\Theta}\left(U, J^{1}\left(\mathbb{R} \times \mathbb{R}^{n}, \mathbb{R}\right)\right)^{(m)}$.

This is proved by an analogous method of [8, p.345 Theorem 6.4].

We denote the ring $\mathcal{E}(1+n+n, m)$ on the coordinates $(t, q, p)$ by $\mathcal{E}_{t, q, p(m)}$ and denote other notations analogously.

THeOREM 4.6. Let $\mathcal{L}=\left(\mathcal{L}_{1}, \ldots, \mathcal{L}_{m}\right)$ be a multi-reticular Legendrian unfolding with a multi-generating family $F=\left(F_{1}, \ldots, F_{m}\right)$. Let $C=\left(C_{1}, \ldots, C_{m}\right) \in C_{T}^{\Theta}\left(J^{1}(\mathbb{R} \times\right.$ $\left.\left.\mathbb{R}^{n}, \mathbb{R}\right), 0\right)^{(m)}$ be an extension of $\mathcal{L}$. Then the following are equivalent.

(u) $F$ is a reticular $t-(\mathcal{P}-\mathcal{K})_{(m)}$-stable unfolding of $\left.F\right|_{t=0}$.

(hs) $\mathcal{L}$ is homotopically stable.

(is) $\mathcal{L}$ is infinitesimally stable.

(a) $\mathcal{E}_{t, q, p(m)}=B_{0}^{r_{1}} \times \cdots \times B_{0}^{r_{m}}+\left\langle 1, p_{1} \circ C^{\prime}, \ldots, p_{n} \circ C^{\prime}\right\rangle_{\left(\Pi \circ C^{\prime}\right)^{*} \mathcal{E}_{t, q, z}}+\left\langle s \circ C^{\prime}\right\rangle_{\mathcal{E}_{t}}$, where $C^{\prime}=\left.C\right|_{z=s=0}, B_{0}^{r_{i}}=\left\langle q_{1} p_{1}, \ldots, q_{r_{i}} p_{r_{i}}, q_{r_{i}+1}, \ldots, q_{n}\right\rangle_{\mathcal{E}_{t, q, p}}$, and $\left(\Pi \circ C^{\prime}\right)^{*} \mathcal{E}_{t, q, z}$ be the $\mathcal{E}_{t, q, z}$-submodule of $\mathcal{E}_{t, q, z(m)}$ such that $\left(\Pi \circ C^{\prime}\right)^{*} a=\left(a\left(\Pi \circ C_{1}^{\prime}\right), \ldots, a\left(\Pi \circ C_{m}^{\prime}\right)\right)$ for $a \in \mathcal{E}_{t, q, z}$.

We remark that sufficiently near multi-reticular Legendrian unfoldings of stable one are also stable by the condition (a).

Proof. $(\mathrm{u}) \Rightarrow(\mathrm{hs})$ : Let $\mathcal{P}$-contact deformations $\left\{C_{\tau}^{i}\right\}$ of $\mathcal{L}_{i}$ be given for $i=1, \ldots, m$. The homotopically stability of multi-reticular Legendrian unfoldings is invariant under $\mathcal{P}_{(m)}$-Legendrian equivalences, we may assume that the map germs

$$
(T, Q, Z, S, P) \rightarrow\left(T, Q, Z, s \circ C_{\tau}^{i}(T, Q, Z, S, P), p \circ C_{\tau}^{i}(T, Q, Z, S, P)\right)
$$

are diffeomorphisms for all $\tau$ and $i$. By Lemma 3.5, there exists a one-parameter families $H_{\tau}^{i}(T, Q, p) \in \mathfrak{M}(1+n+n)^{2}$ depending smoothly on $\tau \in(\mathbb{R}, 0)$ such that the canonical relations $P_{C_{\tau}^{i}}$ associated with $C_{\tau}^{i}$ have the forms (5) for function germs $H_{\tau}^{i}(T, Q, p) \in \mathfrak{M}(1+n+n)^{2}$. We set $\left(0,0,0, s^{i}, p^{i}\right)=\mathcal{L}_{i}(0)$. Then the map germs $F_{\tau}=\left(F_{\tau}^{1}, \ldots, F_{\tau}^{m}\right) \in \mathfrak{M}(r ; n+1+n+1, m)$ defined by

$$
F_{\tau}^{i}(x, y, t, q, z)=-z+H_{\tau}^{i}(t, x, 0, y)+\left\langle y+p^{i}, q\right\rangle+s^{i} t
$$


are generating families of multi-reticular Legendrian unfoldings $\mathcal{L}_{\tau}:=$ $\left(\left.C_{\tau}^{1}\right|_{\mathbb{L}^{r_{1}}}, \ldots,\left.C_{\tau}^{m}\right|_{\mathbb{L}^{r_{m}}}\right)$ for $\tau \in(\mathbb{R}, 0)$. Since $F_{0}$ is a reticular $t$ - $(\mathcal{P}-\mathcal{K})_{(m)}$-stable unfolding of $\left.F\right|_{t=0}$, it follows that $F_{0}$ is a reticular $t$ - $(\mathcal{P}-\mathcal{K})_{(m)}$-homotopically stable unfolding of $\left.F\right|_{t=0}$ by Theorem 2.9. Therefore there exists a one-parameter family of reticular $t-(\mathcal{P}-\mathcal{K})_{(m)}$-isomorphism from $F_{\tau}$ to $F_{0}$ depending smoothly on $\tau$. This means that there exists a one-parameter families of $\mathcal{P}$-Legendrian equivalences $K_{\tau}^{i}$ depending smoothly on $\tau$ such that $K_{\tau}^{i}$ have the forms (7) and

$$
C_{\tau}^{i}\left(L_{\sigma}^{r_{i}, 0}\right)=K_{\tau}^{i} \circ \mathcal{L}\left(L_{\sigma}^{r_{i}, 0}\right) \text { for all } \sigma \subset I_{r}, \tau \in(\mathbb{R}, 0), i \in\{1, \ldots, m\} .
$$

Then the map germs $\Psi_{\tau}^{i}:=\left(C_{0}^{i}\right)^{-1} \circ\left(K_{\tau}^{i}\right)^{-1} \circ C_{\tau}^{i}$ give one-parameter deformations of reticular $r_{i}$-diffeomorphisms on $\left(J^{1}\left(\mathbb{R} \times \mathbb{R}^{n}, \mathbb{R}\right), 0\right)$ and we have that $C_{\tau}^{i}=K_{\tau}^{i} \circ C_{0}^{i} \circ \Psi_{\tau}^{i}$. This means that $\mathcal{L}$ is homotopically stable.

$($ hs $) \Rightarrow($ is $)$ : Let $v=\left(v_{1}, \ldots, v_{m}\right)$ be an infinitesimal $\mathcal{P}$-contact transformation of $\mathcal{L}$. Then there exist $\mathcal{P}$-contact deformations $\left\{C_{\tau}^{i}\right\}$ of $\mathcal{L}_{i}$ such that $v_{i}=\left.\frac{d C_{\tau}^{i}}{d \tau}\right|_{\tau=0}$ for $i=1, \ldots, m$. Then there exist one-parameters of $\mathcal{P}$-Legendrian equivalences $\left\{K_{\tau}^{i}\right\}$ of the form (7) and one-parameter deformations of reticular $\mathcal{P}_{\left(r_{i}\right)}$-diffeomorphisms $\left\{\Psi_{\tau}^{i}\right\}$ such that $C_{\tau}^{i}=K_{\tau}^{i} \circ C_{0}^{i} \circ \Psi_{\tau}^{i}$ for $\tau \in(\mathbb{R}, 0)$ and $i \in\{1, \ldots, m\}$. Then we have that

$$
v_{i}=\left.\frac{d C_{\tau}^{i}}{d \tau}\right|_{\tau=0}=\left.\frac{d K_{\tau}^{i}}{d \tau}\right|_{\tau=0} \circ C_{0}+\left(C_{0}\right)_{*}\left(\left.\frac{d \Psi_{\tau}^{i}}{d \tau}\right|_{\tau=0}\right)
$$

Since $v_{i}$ has the form (9) for each $i$, it follows that $\mathcal{L}$ is infinitesimally stable.

(is) $\Rightarrow\left(\right.$ a): Let a map germ $f=\left(f_{1}, \ldots, f_{m}\right) \in \mathcal{E}_{t, q, p(m)}$ be given. We define the function germs $f_{i}^{\prime}$ on $\left(J^{1}\left(\mathbb{R} \times \mathbb{R}^{n}, \mathbb{R}\right), w_{i}^{\prime}\right)$ by $f_{i}^{\prime}(t, q, z, s, p)=f_{i} \circ \pi_{T, Q, P} \circ$ $C_{i}^{-1}\left(t, q, z, a_{i}(t, q, z, p), p\right)$, where $w_{i}^{\prime}=\left(0, \ldots, 0, p^{i}\right)$ and the function germs $a_{i}$ are defined by $\pi_{S} \circ C_{i}^{-1}\left(t, q, z, a_{i}(t, q, z, p), p\right) \equiv 0$. This equation can be solved by (2). Since $f_{i}^{\prime}$ does not depend on $s$, it follows that $X_{f_{i}^{\prime}} \circ C_{i}$ is an infinitesimal $\mathcal{P}$-contact transformation of $C_{i}$. Therefore there exist an infinitesimal $\mathcal{P}$-Legendrian equivalence $\eta_{i}$ of the form (9) and an infinitesimal reticular $r_{i}$-diffeomorphism $\xi_{i}$ such that $X_{f_{i}^{\prime}} \circ C_{i}=\left(C_{i}\right)_{*} \xi_{i}+\eta_{i} \circ C_{i}$. By Lemma 4.4, there exist a $\mathcal{P}$-fiber preserving function germ $H$ on $\left(J^{1}\left(\mathbb{R} \times \mathbb{R}^{n}, \mathbb{R}\right), 0\right)$ and $g_{i} \in B^{r_{i}}$ such that $\xi_{i}=X_{g_{i}}$ and $\eta_{i}=X_{H}$. Then we have that $f_{i}^{\prime} \circ C_{i}=g_{i}+H \circ C_{i}$. Since $f_{i}^{\prime} \circ C(T, Q, Z, S, P)=$ $f_{i} \circ \pi_{T, Q, P} \circ\left(C_{i}\right)^{-1}\left(t, q, z, a_{i}(T, Q, Z, 0, P), p\right)=f_{i} \circ \pi_{T, Q, P}(T, Q, Z, 0, P)=f_{i}(T, Q, P)$ and $H$ has the form $H(t, q, z, s, p)=\sum_{j=1}^{n} h_{j}(t, q, z) p_{i}+h_{0}(t, q, z)+h^{\prime}(t) s$, We have that

$$
f_{i} \equiv \sum_{j=1}^{n}\left(h_{j}\left(\Pi \circ C_{i}^{\prime}\right)\right)\left(p_{i} \circ C_{i}^{\prime}\right)+h_{0}\left(\Pi \circ C_{i}^{\prime}\right)+\left(h^{\prime}\left(t \circ C_{i}^{\prime}\right)\right)\left(s \circ C_{i}^{\prime}\right) \bmod B_{0}^{r_{i}} .
$$

Since $t \circ C_{i}=t$, we have the required form.

$(\mathrm{a}) \Rightarrow(\mathrm{u})$ : By Lemma 3.5, there exists function germs $H_{i}(T, Q, p) \in \mathfrak{M}(1+n+n)^{2}$ such that the function germ $H_{i}(T, Q, p)-T s$ is a generating function of $P_{C_{i}}$ for each $i$. Then the map germ $F=\left(F_{1}, \ldots, F_{m}\right) \in \mathfrak{M}(r ; n+1+n+1, m)$ given by $F_{i}(x, y, t, q, z)=-z+H_{i}(t, x, 0, y)+s^{i} t+\left\langle y+p^{i}, q\right\rangle$ is a generating family of $\mathcal{L}$. Then $P_{C_{i}}^{\prime}:=P_{C_{i}} \mid Z=S=0$ has the form

$$
P_{C_{i}}^{\prime}=\left\{\left(T, Q,-\frac{\partial H_{i}}{\partial Q}, T,-\frac{\partial H_{i}}{\partial p}, H_{i}-\left\langle\frac{\partial H_{i}}{\partial p}, p+p^{i}\right\rangle+s^{i} T, \frac{\partial H_{i}}{\partial T}+s^{i}, p+p^{i}\right)\right\} .
$$

Then the map germ $P_{C_{i}}^{\prime} \rightarrow\left(\mathbb{R}^{1+n+n}, 0\right), w \mapsto \pi_{T, Q, P}(w)$ is a diffeomorphism. We set $D\left(F_{i}\right)=\left\{(x, y, t, q, z) \in\left(\mathbb{H}^{r} \times \mathbb{R}^{n+1+n+1}, 0\right) \mid F_{i}=x \frac{\partial F_{i}}{\partial x}=\frac{\partial F_{i}}{\partial y}=0\right\}$. We also define 
the map germ $D\left(F_{i}\right) \rightarrow P_{C_{i}}^{\prime}$ by

$$
(x, y, t, q, z) \mapsto\left(t, x, 0,-\frac{\partial F_{i}}{\partial x},-\frac{\partial H_{i}}{\partial Q_{r+1}}(t, x, 0, y), \ldots,-\frac{\partial H_{i}}{\partial Q_{n}}, t, q, z, \frac{\partial F_{i}}{\partial t}, y+p^{i}\right) .
$$

Then the composition of the above two map germs induces the map germ $\mathcal{E}_{T, Q, P} / B_{0}^{r_{i}} \rightarrow \mathcal{E}_{D\left(F_{i}\right)}$. We denote $T, Q, P$ for the variables on the source space of this map germ. Then the correspondence is given that:

$$
\begin{gathered}
T \mapsto t, Q_{1} \mapsto x_{1}, \ldots, Q_{r} \mapsto x_{r}, P_{1} \mapsto-\frac{\partial F_{i}}{\partial x_{1}}, \ldots, P_{r} \mapsto-\frac{\partial F i}{\partial x_{r}}, \\
t \circ C_{i}^{\prime}(T, Q, P) \mapsto t, q \circ C^{\prime} i(T, Q, P) \mapsto q, z \circ C_{i}^{\prime}(T, Q, P) \mapsto z, \\
s \circ C_{i}^{\prime}(T, Q, P) \mapsto \frac{\partial F_{i}}{\partial t}, p \circ C_{i}^{\prime}(T, Q, P) \mapsto y+p^{i},\left(\left(\Pi \circ C_{i}^{\prime}\right)^{*} \mathcal{E}_{t, q, z}\right) \mapsto \mathcal{E}_{t, q, z},\left(\left(t \circ C_{i}^{\prime}\right)^{*} \mathcal{E}_{t}\right) \mapsto \mathcal{E}_{t} .
\end{gathered}
$$

Then (a) is transferred that

$$
\begin{aligned}
& \mathcal{E}(r ; n+1+n+1)=\left\langle F, x \frac{\partial F}{\partial x}, \frac{\partial F}{\partial y}\right\rangle_{\mathcal{E}(r ; n+1+n+1)} \\
& \quad+\left\langle 1^{(m)}\left(=-\frac{\partial F}{\partial z}\right),\left(y_{1}+p_{1}\right)^{(m)}\left(=\frac{\partial F}{\partial q_{1}}\right), \ldots,\left(y_{n}+p_{n}\right)^{(m)}\left(=\frac{\partial F}{\partial q_{n}}\right)\right\rangle_{\mathcal{E}_{t, q, z}}+\left\langle\frac{\partial F}{\partial t}\right\rangle_{\mathcal{E}_{t}},
\end{aligned}
$$

where $a^{(m)}=(a, \ldots, a)$ for each function germ $a$ and $\left(y_{i}+p_{i}\right)^{(m)}=\left(y_{i}+p_{i}^{1}, \ldots, y_{i}+\right.$ $\left.p_{i}^{n}\right)$. It follows that $F$ is a reticular $t$ - $\mathcal{P}$ - $\mathcal{K}$-infinitesimal stable unfolding of $\left.F\right|_{t=0}$. We have that $F$ is a reticular $t$ - $\mathcal{P}$ - $\mathcal{K}$-stable unfolding of $\left.F\right|_{t=0}$ by Theorem 2.9.

5. Genericity. In order to give a generic classification of multi-reticular Legendrian unfoldings, we reduce our investigation to finite dimensional jet spaces of $\mathcal{P}$-contact diffeomorphism germs.

Definition 5.1. Let $\mathcal{L}=\left(\mathcal{L}_{1}, \ldots, \mathcal{L}_{m}\right)$ be a multi-reticular Legendrian unfolding. We say that $\mathcal{L}$ is $l$-determined if the following condition holds: For any extension $C_{i} \in C_{T}\left(J^{1}\left(\mathbb{R} \times \mathbb{R}^{n}, \mathbb{R}\right), 0\right)$ of $\mathcal{L}_{i}$, the multi-reticular Legendrian unfolding $\left(\left.C_{1}^{\prime}\right|_{\mathbb{L}^{r_{1}}}, \ldots,\left.C_{m}^{\prime}\right|_{\mathbb{L}^{r_{m}}}\right)$ and $\mathcal{L}$ are $\mathcal{P}_{(m)}$-Legendrian equivalent for all $C_{i}^{\prime} \in$ $C_{T}\left(J^{1}\left(\mathbb{R} \times \mathbb{R}^{n}, \mathbb{R}\right), 0\right)$ satisfying that $j^{l} C_{i}(0)=j^{l} C_{i}^{\prime}(0)$ for $i=1, \ldots, m$.

As Lemma 4.3, we may consider the following other definition of finitely determinacy of reticular Legendrian maps:

(1) The definition given by replacing $C_{T}\left(J^{1}\left(\mathbb{R} \times \mathbb{R}^{n}, \mathbb{R}\right), 0\right)$ to $C_{T}^{\Theta}\left(J^{1}\left(\mathbb{R} \times \mathbb{R}^{n}, \mathbb{R}\right), 0\right)$.

(2) The definition given by replacing $C_{T}\left(J^{1}\left(\mathbb{R} \times \mathbb{R}^{n}, \mathbb{R}\right), 0\right)$ to $C_{T}^{Z}\left(J^{1}\left(\mathbb{R} \times \mathbb{R}^{n}, \mathbb{R}\right), 0\right)$.

(3) The definition given by replacing $C_{T}\left(J^{1}\left(\mathbb{R} \times \mathbb{R}^{n}, \mathbb{R}\right), 0\right)$ to $C_{T}^{\Theta, Z}\left(J^{1}\left(\mathbb{R} \times \mathbb{R}^{n}, \mathbb{R}\right), 0\right)$. Then the following holds by [8, p.341 Proposition 5.6]:

Proposition 5.2. Let $\mathcal{L}$ be a multi-reticular Legendrian unfolding. Then (A) If $\mathcal{L}$ is l-determined of the original definition, then $\mathcal{L}$ is l-determined of the definition (1).

(B) If $\mathcal{L}$ is l-determined of the definition (1), then $\mathcal{L}$ is l-determined of the definition (3).

(C) If $\mathcal{L}$ is $(l+1)$-determined of the definition $(3)$, then $\mathcal{L}$ is $l$-determined of the definition (2).

(D) If $\mathcal{L}$ is 1 -determined of the definition (2), then $\mathcal{L}$ is l-determined of the original definition. 
THEOREM 5.3. Let $\mathcal{L}=\left(\mathcal{L}_{1}, \ldots, \mathcal{L}_{m}\right)$ be a multi reticular Legendrian unfolding. If $\mathcal{L}$ is infinitesimally stable then $\mathcal{L}$ is $(n+5)$-determined.

Proof. It is enough to prove $\mathcal{L}$ is $(n+4)$-determined of Definition 5.1 (3). Let $C_{i} \in C_{T}^{\Theta, Z}\left(J^{1}\left(\mathbb{R} \times \mathbb{R}^{n}, \mathbb{R}\right), 0\right)$ be extensions of $\mathcal{L}_{i}$ for $i=1, \ldots, m$. Since the finite determinacy of multi-reticular Legendrian unfoldings is invariant under $\mathcal{P}_{(m)}$-Legendrian equivalences, we may assume that $P_{C_{i}}$ have the form (5) for some function germs $H_{i}(T, Q, p) \in \mathfrak{M}(2 n+1)^{2}$. Then $F_{i}(x, y, t, q, z)=-z+H_{0, i}(x, y, t)+\left\langle y+p^{i}, q\right\rangle \in$ $\mathfrak{M}(r ; n+1+n+1)$ is a generating family of $\mathcal{L}_{i}$, where $\left(0,0,0, s^{i}, p^{i}\right)=\mathcal{L}_{i}(0)$ and $H_{0, i}(x, y, t)=H_{i}(t, x, 0, y)+s^{i} t \in \mathfrak{M}(r ; n+1)$.

By Theorem 4.6, we have that $F=\left(F_{1}, \ldots, F_{m}\right)$ is a reticular $t-(\mathcal{P}-\mathcal{K})_{(m)^{-}}$ infinitesimally versal unfolding of $f:=\left.F\right|_{t=0}$. It follows that

$$
\begin{gathered}
\mathcal{E}(r ; n+1+n+1, m)=\left\langle F_{1}, x \frac{\partial F_{1}}{\partial x}, \frac{\partial F_{1}}{\partial y}\right\rangle_{\mathcal{E}(r ; n+1+n+1)} \times \cdots \times \\
\left\langle F_{m}, x \frac{\partial F_{m}}{\partial x}, \frac{\partial F_{m}}{\partial y}\right\rangle_{\mathcal{E}(r ; n+1+n+1)}+\left\langle\frac{\partial F}{\partial q}, \frac{\partial F}{\partial z}\right\rangle_{\mathcal{E}(1+n+1)}+\left\langle\frac{\partial F}{\partial t}\right\rangle_{\mathcal{E}(1)}
\end{gathered}
$$

By the restriction of the both side to $q=z=0$, we have that

$$
\begin{aligned}
& \mathcal{E}(r ; n+1, m)= \\
& \left\langle H_{0,1}, x \frac{\partial H_{0,1}}{\partial x}, \frac{\partial H_{0,1}}{\partial y}\right\rangle_{\mathcal{E}(r ; n+1)} \times \cdots \times\left\langle H_{0, m}, x \frac{\partial H_{0, m}}{\partial x}, \frac{\partial H_{0, m}}{\partial y}\right\rangle_{\mathcal{E}(r ; n+1)} \\
& +\left\langle 1^{(m)},\left(y_{1}+p_{1}\right)^{(m)}, \ldots,\left(y_{n}+p_{n}\right)^{(m)}, \frac{\partial H_{0}}{\partial t}\right\rangle_{\mathcal{E}(1)}
\end{aligned}
$$

where $H_{0}=\left(H_{0,1}, \ldots, H_{0, m}\right)$. It follows that

$$
\begin{aligned}
& \mathfrak{M}(r ; n+1, m)^{n+2} \subset \\
& \left\langle H_{0,1}, x \frac{\partial H_{0,1}}{\partial x}, \frac{\partial H_{0,1}}{\partial y}\right\rangle_{\mathcal{E}(r ; n+1)} \times \cdots \times\left\langle H_{0, m}, x \frac{\partial H_{0, m}}{\partial x}, \frac{\partial H_{0, m}}{\partial y}\right\rangle_{\mathcal{E}(r ; n+1)} \\
& +\mathfrak{M}(1) \mathcal{E}(r ; n+1, m) .
\end{aligned}
$$

By (10) and (11) we have that

$$
\begin{aligned}
\mathfrak{M}(r ; n+1, m)^{n+3} \subset\left(\left\langleH_{0,1},\right.\right. & \left.\left.x \frac{\partial H_{0,1}}{\partial x}\right\rangle_{\mathcal{E}(r ; n+1)}+\mathfrak{M}(r ; n+1)\left\langle\frac{\partial H_{0,1}}{\partial y}\right\rangle\right) \times \cdots \times \\
& \left(\left\langle H_{0, m}, x \frac{\partial H_{0, m}}{\partial x}\right\rangle_{\mathcal{E}(r ; n+1)}+\mathfrak{M}(r ; n+1)\left\langle\frac{\partial H_{0, m}}{\partial y}\right\rangle\right) \\
+ & \mathfrak{M}(1)\left\langle 1^{(m)},\left(y_{1}+p_{1}\right)^{(m)}, \ldots,\left(y_{n}+p_{n}\right)^{(m)}, \frac{\partial H_{0}}{\partial t}\right\rangle .
\end{aligned}
$$

Let $C_{i}^{\prime} \in C_{T}^{\Theta, Z}\left(J^{1}\left(\mathbb{R} \times \mathbb{R}^{n}, \mathbb{R}\right), 0\right)$ satisfying $j^{n+4} C_{i}(0)=j^{n+4} C_{i}^{\prime}(0)$ be given for $i=$ $1, \ldots, m$. There exist function germs $H_{i}^{\prime}(T, Q, p) \in \mathfrak{M}(2 n+1)^{2}$ such that

$$
\begin{aligned}
P_{C_{i}^{\prime}}=\{(T, Q,- & \frac{\partial H_{i}^{\prime}}{\partial T}(T, Q, p)+s,-\frac{\partial H_{i}^{\prime}}{\partial Q}, T,-\frac{\partial H_{i}^{\prime}}{\partial p}, \\
& \left.\left.H_{i}^{\prime}-\left\langle\frac{\partial H_{i}^{\prime}}{\partial p}, p+p^{i}\right\rangle+s^{i} T, s+s^{i}, p+p^{i}\right)\right\} .
\end{aligned}
$$

Since $H_{i}=z-q p$ on $P_{C_{i}}$ and $H_{i}^{\prime}=z-q p$ on $P_{C_{i}^{\prime}}$, we have that $j^{n+4} H_{0, i}(0)=$ $j^{n+4} H_{0, i}^{\prime}(0)$, where $H_{0, i}^{\prime}(x, y, t)=H_{i}^{\prime}(t, x, 0, y)+s^{i} t \in \mathfrak{M}(r ; n+1)$. By (11) we have 
that

$$
\begin{aligned}
\mathfrak{M}(r ; n, m)^{n+2} & \subset\left\langle H_{0,1}(x, y, 0), x \frac{\partial H_{0,1}}{\partial x}(x, y, 0), \frac{\partial H_{0,1}}{\partial y}(x, y, 0)\right\rangle_{\mathcal{E}(r ; n)} \\
& \times \cdots \times\left\langle H_{0, m}(x, y, 0), x \frac{\partial H_{0, m}}{\partial x}(x, y, 0), \frac{\partial H_{0, m}}{\partial y}(x, y, 0)\right\rangle_{\mathcal{E}(r ; n)}
\end{aligned}
$$

and this means that $H_{0, i}(x, y, 0)$ is reticular $\mathcal{K}$ - $(n+3)$-determined by Lemma 2.3 and hence $H_{0}(x, y, 0)$ is also reticular $\mathcal{K}_{(m)^{-}}(n+3)$-determined. So we may assume that $H_{0}-H_{0}^{\prime} \in \mathfrak{M}(1) \mathfrak{M}(r ; n+1, m)^{n+3}$. Then the function germs

$$
G_{i}(x, y, t, q, z)=-z+H_{0, i}^{\prime}(x, y, t)+\left\langle y+p^{i}, q\right\rangle \in \mathfrak{M}(r ; n+1+n+1)
$$

are generating families of $\left.C_{i}^{\prime}\right|_{\mathbb{L}^{r_{i}}}$ for $i=1, \ldots, m$.

We define the function germs $E_{\tau_{0}, i}(x, y, t, \tau) \in \mathcal{E}(r ; n+1+1)$ by $E_{\tau_{0}, i}(x, y, t, \tau)=$ $\left(1-\tau-\tau_{0}\right) H_{0, i}(x, y, t)+\left(\tau+\tau_{0}\right) H_{0, i}^{\prime}(x, y, t)$ for $\tau_{0} \in[0,1]$ and define $E_{\tau_{0}}(x, y, t, \tau) \in$ $\mathcal{E}(r ; n+1+1, m)$ by $E_{\tau_{0}}=\left(E_{\tau_{0}, 1}, \ldots, E_{\tau_{0}, m}\right)$ By an analogous method of the proof of $[7$, Lemma 5.10], we have that

$$
\begin{array}{r}
\frac{\partial E_{\tau_{0}}}{\partial \tau} \in \mathfrak{M}_{t}\left(\left\langle E_{\tau_{0}, 1}, x \frac{\partial E_{\tau_{0}, 1}}{\partial x}\right\rangle_{\mathcal{E}(r ; n+2)}+\mathfrak{M}(r ; n+2)\left\langle\frac{\partial E_{\tau_{0}, 1}}{\partial y}\right\rangle\right) \times \cdots \times \\
\left(\left\langle E_{\tau_{0}, m}, x \frac{\partial E_{\tau_{0}, m}}{\partial x}\right\rangle_{\mathcal{E}(r ; n+2)}+\mathfrak{M}(r ; n+2)\left\langle\frac{\partial E_{\tau_{0}, m}}{\partial y}\right\rangle\right) \\
+\mathfrak{M}_{t}^{2}\left\langle 1^{(m)},\left(y_{1}+p_{1}\right)^{(m)}, \ldots,\left(y_{n}+p_{n}\right)^{(m)}, \frac{\partial E_{\tau_{0}}}{\partial t}\right\rangle
\end{array}
$$

for $\tau_{0} \in[0,1]$. This means that there exist $\Phi_{i}(x, y, t) \in \mathcal{B}_{1}(r ; n+1)$ and units $a_{i} \in$ $\mathcal{E}(r ; n+1)$ and $b_{1}(t), \ldots, b_{n}(t), c(t) \in \mathfrak{M}(1)^{2}$ such that

(1) $\Phi_{i}$ has the form

$$
\Phi_{i}(x, y, t)=\left(x \phi_{1}^{i}(x, y, t, q, z), \phi_{2}^{i}(x, y, t, q, z), \phi_{3}(t)\right)
$$

(2) $H_{0, i}^{\prime}(x, y, t)=a_{i}(x, y, t) \cdot H_{0, i} \circ \Phi_{i}(x, y, t)+\sum_{j=1}^{n}\left(y_{j}+p_{j}^{i}\right) b_{j}(t)+c(t)$ for $(x, y, t) \in \mathbb{H}^{r} \times \mathbb{R}^{n+1}$ and $i=1, \ldots, m$,

(3) $\phi(x, y, 0)=(x, y), a(x, y)=1$ for $(x, y) \in \mathbb{H}^{r} \times \mathbb{R}^{n}$.

This means that $F$ and $G=\left(G_{1}, \ldots, G_{m}\right)$ are reticular $t-(\mathcal{P}-\mathcal{K})_{(m)}$-infinitesimal versal unfoldings of $f$. It follows that $F$ and $G$ are reticular $t$ - $(\mathcal{P}-\mathcal{K})_{(m)}$-equivalent. Therefore $\mathcal{L}$ and $\left(\left.C_{1}^{\prime}\right|_{\mathbb{L}^{r_{1}}}, \ldots,\left.C_{m}^{\prime}\right|_{\mathbb{L}^{r_{m}}}\right)$ are $\mathcal{P}_{(m)}$-Legendrian equivalent. $\mathrm{Z}$

Let $J^{l}(2 n+3,2 n+3)^{(m)}$ be the set of multi-l-jets of map germs from $\left(J^{1}(\mathbb{R} \times\right.$ $\left.\left.\mathbb{R}^{n}, \mathbb{R}\right), 0\right)$ to $J^{1}\left(\mathbb{R} \times \mathbb{R}^{n}, \mathbb{R}\right)$ and $t C^{l}(n, m)$ be the immersed manifold in $J^{l}(2 n+3,2 n+$ $3)^{(m)}$ which consists of multi- $l$-jets of $\mathcal{P}$-contact embedding germs. Let $L^{l}(2 n+3)^{(m)}$ be the Lie group which consists of multi-l-jets of diffeomorphism germs on $\left(J^{1}(\mathbb{R} \times\right.$ $\left.\left.\mathbb{R}^{n}, \mathbb{R}\right), 0\right)$.

We consider the Lie subgroup $r t L e^{l}(n, m)$ of $L^{l}(2 n+3)^{(m)} \times L^{l}(2 n+3)$ which consists of multi- $l$-jets of reticular $r_{i}$-diffeomorphisms on the source space and $l$-jets of $\mathcal{P}$-Legendrian equivalences of $\Pi$ at 0 :

$$
\begin{array}{r}
r t L e^{l}(n, m)=\left\{\left(j^{l} \Psi_{1}(0), \ldots, j^{l} \Psi_{m}(0), j^{l} K(0)\right) \in L^{l}(2 n+3)^{(m)} \times L^{l}(2 n+3) \mid\right. \\
\Psi_{i} \text { is a reticular } r_{i} \text {-diffeomorphism on }\left(J^{1}\left(\mathbb{R} \times \mathbb{R}^{n}, \mathbb{R}\right), 0\right), \\
K \text { is a } \mathcal{P} \text {-Legendrian equivalence of } \Pi\} .
\end{array}
$$


The group $r t L e^{l}(n, m)$ acts on $J^{l}(2 n+3,2 n+3)$ and $t C^{l}(n, m)$ is invariant under this action. Let $C=\left(C_{1}, \ldots, C_{m}\right)$ be a $\mathcal{P}_{(m)}$-contact diffeomorphism germ from $\left(J^{1}\left(\mathbb{R} \times \mathbb{R}^{n}, \mathbb{R}\right), 0\right)$ to $J^{1}\left(\mathbb{R} \times \mathbb{R}^{n}, \mathbb{R}\right)$ and set $z_{i}=j^{l} C_{i}(0), \quad \mathcal{L}_{i}=\left.C_{i}\right|_{\mathbb{L}^{r_{i}}}$, $\mathcal{L}=\left(\mathcal{L}_{1}, \ldots, \mathcal{L}_{m}\right), z=\left(z_{1}, \ldots, z_{m},\right)$. We denote the orbit $\operatorname{rtLe}^{l}(n, m) \cdot z$ by $[z]$. Then

$$
[z]=\left\{j^{l} C^{\prime}(0) \in t C^{l}(n, m) \mid \mathcal{L} \text { and }\left(\left.C_{1}^{\prime}\right|_{\mathbb{L}^{r_{1}}}, \ldots,\left.C_{m}^{\prime}\right|_{\mathbb{L}^{r_{m}}}\right) \text { are } \mathcal{P}_{(m)} \text {-Legendrian equivalent }\right\}
$$

We denote by $V I_{C}$ the vector space consisting of infinitesimal $\mathcal{P}_{(m)}$-contact transformation germs of $C$ and denote by $V I_{C}^{0}$ the subspace of $V I_{C}$ consist of germs which vanish on 0 . We denote by $V L_{J^{1}\left(\mathbb{R} \times \mathbb{R}^{n}, \mathbb{R}\right)}$ by the vector space consisting of infinitesimal $\mathcal{P}$-Legendrian equivalences on $\Pi$ and denote by $V L_{J^{1}\left(\mathbb{R} \times \mathbb{R}^{n}, \mathbb{R}\right)}^{0}$ by the subspace of $V L_{J^{1}\left(\mathbb{R} \times \mathbb{R}^{n}, \mathbb{R}\right)}$ consists of germs which vanish at 0 .

We denote by $V_{\mathbb{L}^{r}}^{0}$ the vector space consisting of infinitesimal reticular $r$ diffeomorphisms on $\left(J^{1}\left(\mathbb{R} \times \mathbb{R}^{n}, \mathbb{R}\right), 0\right)$ which vanishes at 0 and set $V_{\mathbb{L}}^{0}=V_{\mathbb{L}^{r_{1}}}^{0} \times V_{\mathbb{L}^{r_{m}}}^{0}$. We have that by Lemma 4.4:

$$
\begin{gathered}
V I_{C}^{0}=\left\{\left(v_{1}, \ldots, v_{m}\right) \mid v_{i}:\left(J^{1}\left(\mathbb{R} \times \mathbb{R}^{n}, \mathbb{R}\right), 0\right) \rightarrow T\left(J^{1}\left(\mathbb{R} \times \mathbb{R}^{n}, \mathbb{R}\right)\right),\right. \\
\left.v_{i}=X_{f_{i}} \circ C_{i} \text { for some } f \in \mathfrak{M}_{t, q, z, p}^{2}\right\}, \\
V L_{J^{1}\left(\mathbb{R} \times \mathbb{R}^{n}, \mathbb{R}\right)}^{0}=\left\{\eta \in X\left(J^{1}\left(\mathbb{R} \times \mathbb{R}^{n}, \mathbb{R}\right), 0\right) \mid \eta=X_{H}\right. \text { for some } \\
\left.\mathcal{P} \text {-fiver preserving function germ } H \in \mathfrak{M}_{J^{1}\left(\mathbb{R} \times \mathbb{R}^{n}, \mathbb{R}\right)}^{2}\right\}, \\
V_{\mathbb{L}}^{0}=\left\{\left(\xi_{1}, \ldots, \xi_{m}\right) \mid \xi_{i} \in X\left(J^{1}\left(\mathbb{R} \times \mathbb{R}^{n}, \mathbb{R}\right), 0\right), \xi_{i}=X_{g_{i}} \text { for some } g_{i} \in B^{r_{i}} \cap \mathfrak{M}_{J^{1}\left(\mathbb{R} \times \mathbb{R}^{n}, \mathbb{R}\right)}^{2}\right\} .
\end{gathered}
$$

We define the homomorphism $t C: \mathfrak{M}_{J^{1}\left(\mathbb{R} \times \mathbb{R}^{n}, \mathbb{R}\right)} V I_{\mathbb{L}} \rightarrow V I_{C}^{0}$ by $t C(v)=\left(C_{1 *} v_{1}\right.$, $\left.\ldots, C_{m *} v_{m}\right)$ and define the homomorphism $w C: V L_{J^{1}\left(\mathbb{R} \times \mathbb{R}^{n}, \mathbb{R}\right)}^{0} \rightarrow V I_{C}^{0}$ by $w C(\eta)=$ $\left(\eta \circ C_{1}, \ldots, \eta \circ C_{m}\right)$.

We denote $V I_{C}^{l}$ the subspace of $V I_{C}$ consisting of infinitesimal $\mathcal{P}$-contact transformation germs of $C$ whose $l$-jets are 0 :

$$
V I_{C}^{l}=\left\{\left(v_{1}, \ldots, v_{m}\right) \in V I_{C} \mid j^{l} v_{i}(0)=0\right\} .
$$

For $\tilde{C}=\left(\tilde{C}_{1}, \ldots, \tilde{C}_{m}\right) \in C_{T}\left(U, J^{1}\left(\mathbb{R} \times \mathbb{R}^{n}, \mathbb{R}\right)\right)^{(m)}$, we define the continuous map $j_{0}^{l} \tilde{C}: U \rightarrow t C^{l}(n, m)$ by $x$ to the $l$-jet of $\left(\tilde{C}_{1 x}, \ldots, \tilde{C}_{m x}\right)$.

TheOREM 5.4. Let $\mathcal{L}=\left(\mathcal{L}_{1}, \ldots, \mathcal{L}_{m}\right)$ be a reticular Legendrian unfolding. Let $C_{i}$ be an extension of $\mathcal{L}_{i}$ and $l \geq(n+2)^{2}$. We set $C=\left(C_{1}, \ldots, C_{m}\right)$. Then the followings are equivalent:

(s) $\mathcal{L}$ is stable.

(t) $j_{0}^{l} C$ is transversal to $\left[j_{0}^{l} C(0)\right]$.

(a') $\mathcal{E}_{t, q, p(m)}=B_{0}^{r_{1}} \times \cdots \times B_{0}^{r_{m}}+\left\langle 1, p_{1} \circ C^{\prime}, \ldots, p_{n} \circ C^{\prime}\right\rangle_{\left(\Pi \circ C^{\prime}\right)^{*} \mathcal{E}_{t, q, z}}+\left\langle s \circ C^{\prime}\right\rangle_{\mathcal{E}_{t}}+$ $\mathfrak{M}_{t, q, p(m)}^{l}$, where $C^{\prime}=\left.C\right|_{z=s=0}$ and $B_{0}^{r_{i}}=\left\langle q_{1} p_{1}, \ldots, q_{r_{i}} p_{r_{i}}, q_{r_{i}+1}, \ldots, q_{n}\right\rangle_{\mathcal{E}_{t, q, p}}$,

(a) $\mathcal{E}_{t, q, p(m)}=B_{0}^{r_{1}} \times \cdots \times B_{0}^{r_{m}}+\left\langle 1, p_{1} \circ C^{\prime}, \ldots, p_{n} \circ C^{\prime}\right\rangle_{\left(\Pi \circ C^{\prime}\right)^{*} \mathcal{E}_{t, q, z}}+\left\langle s \circ C^{\prime}\right\rangle_{\mathcal{E}_{t}}$,

(is) $\mathcal{L}$ is infinitesimally stable,

(hs) $\mathcal{L}$ is homotopically stable,

(u) A multi-generating family $F$ of $\mathcal{L}$ is reticular $t-(\mathcal{P}-\mathcal{K})_{(m)}$-stable unfolding of $\left.F\right|_{t=0}$.

Proof. $(\mathrm{s}) \Rightarrow(\mathrm{t})$ : By theorem 4.5 and $(\mathrm{s})$, there exists a multi- $\mathcal{P}$-contact embedding $\tilde{C}^{\prime}=\left(\tilde{C}_{1}^{\prime}, \ldots, \tilde{C}_{m}^{\prime}\right)$ around $\tilde{C}$ such that $j_{0}^{l} \tilde{C}^{\prime}$ is transversal to $\left[j_{0}^{l} C(0)\right]$, 
and $\left(\left.\left(\tilde{C}_{1}^{\prime}\right)_{x_{1}}\right|_{\mathbb{L}^{r_{1}}}, \ldots,\left.\left(\tilde{C}_{m}^{\prime}\right)_{x_{m}}\right|_{\mathbb{L}^{r_{m}}}\right)$ and $\mathcal{L}$ are $\mathcal{P}_{(m)}$-Legendrian equivalent for $x_{i}=$ $\left(t^{i}, 0, \ldots, 0, p_{r+1}^{i}, \ldots, p_{n}^{i}\right) \in U$. This means that $\left[j_{0}^{l} \tilde{C}_{x}^{\prime}(0)\right]=\left[j_{0}^{l} C(0)\right]$ and hence $j_{0}^{l} C$ is transversal to $\left[j_{0}^{l} C(0)\right]$ at 0 for $x=\left(x_{1}, \ldots, x_{m}\right)$.

$(\mathrm{t}) \Leftrightarrow(\mathrm{a})$ : This is proved by an analogous method of Theorem 4.6.

(a) $\Leftrightarrow\left(a^{\prime}\right)$ : We need only to prove $\left(a^{\prime}\right) \Rightarrow(a)$. By the restriction of (a') to $t=0$ we have that:

$\mathcal{E}_{q, p(m)}=B_{1}^{r_{1}} \times \cdots \times B_{1}^{r_{m}}+\left\langle 1, p_{1} \circ C^{\prime \prime}, \ldots, p_{n} \circ C^{\prime \prime}\right\rangle_{\left(\Pi \circ C^{\prime \prime}\right)^{*} \mathcal{E}_{t, q, z}}+\left\langle s \circ C^{\prime \prime}\right\rangle_{\mathbb{R}}+\mathfrak{M}_{q, p(m)}^{l}$,

where $C^{\prime \prime}=\left.C^{\prime}\right|_{t=0}$ and $B_{1}^{r_{i}}=\left.B_{0}^{r_{i}}\right|_{t=0}$. Then we have that

$\mathcal{E}_{q, p(m)}=B_{1}^{r_{1}} \times \cdots \times B_{1}^{r_{m}}+\left(\Pi \circ C^{\prime \prime}\right)^{*} \mathfrak{M}_{t, q, p} \mathcal{E}_{q, p(m)}+\left\langle 1, p_{1} \circ C^{\prime \prime}, \ldots, p_{n} \circ C^{\prime \prime}, s \circ C^{\prime \prime}\right\rangle_{\mathbb{R}}+\mathfrak{M}_{q, p(m)}^{l}$.

It follows that

$$
\mathfrak{M}_{q, p(m)}^{n+2} \subset B_{1}^{r_{1}} \times \cdots \times B_{1}^{r_{m}}+\left(\Pi \circ C^{\prime \prime}\right)^{*} \mathfrak{M}_{t, q, p} \mathcal{E}_{q, p(m)} .
$$

Therefore

$$
\mathfrak{M}_{t, q, p(m)}^{n+2} \subset B_{0}^{r_{1}} \times \cdots \times B_{0}^{r_{m}}+\left(\Pi \circ C^{\prime}\right)^{*} \mathfrak{M}_{t, q, p} \mathcal{E}_{q, p(m)}+\mathfrak{M}_{t} \mathcal{E}_{t, q, p(m)},
$$

and we have that

$\mathfrak{M}_{t, q, p(m)}^{l}=\left(\mathfrak{M}_{t, q, p(m)}^{n+2}\right)^{n+2} \subset B_{0}^{r_{1}} \times \cdots \times B_{0}^{r_{m}}+\left(\Pi \circ C^{\prime}\right)^{*} \mathfrak{M}_{t, q, p}^{n+2} \mathcal{E}_{t, q, p(m)}+\mathfrak{M}_{t} \mathcal{E}_{t, q, p(m)}$.

It follows that

$$
\begin{aligned}
\mathcal{E}_{t, q, p(m)}=B_{0}^{r_{1}} & \times \cdots \times B_{0}^{r_{m}}+\left\langle 1, p_{1} \circ C^{\prime}, \ldots, p_{n} \circ C^{\prime}\right\rangle_{\left(\Pi \circ C^{\prime}\right)^{*} \mathcal{E}_{t, q, z}} \\
& +\left\langle s \circ C^{\prime}\right\rangle_{\mathcal{E}_{t}}+\left(\Pi \circ C^{\prime}\right)^{*} \mathfrak{M}_{t, q, p}^{n+2} \mathcal{E}_{t, q, p(m)}+\mathfrak{M}_{t} \mathcal{E}_{t, q, p(m)} .
\end{aligned}
$$

This means (a) by Lemma 2.1 .

(a) $\Leftrightarrow($ is $) \Leftrightarrow(\mathrm{hs}) \Leftrightarrow(\mathrm{u})$ : This is proved in Theorem 4.6.

(t) $\&($ is $) \Rightarrow(\mathrm{s})$ : Since $j_{0}^{l} C$ is transversal to $\left[j_{0}^{l} C(0)\right]$, it follows that there exists a neighborhood $W_{\tilde{C}}$ of $\tilde{C}$ in $C_{T}^{\Theta}\left(U, J^{1}\left(\mathbb{R} \times \mathbb{R}^{n}, \mathbb{R}\right)\right)^{(m)}$ such that for any $\tilde{C}^{\prime} \in W_{\tilde{C}}$ there exists $x \in U^{(m)}$ such that $j_{0}^{l} \tilde{C}^{\prime}$ is transversal to $\left[j_{0}^{l} C(0)\right]$ at $x$. Since $j_{0}^{l} \tilde{C}_{x}^{\prime} \in\left[j_{0}^{l} C(0)\right]$, it follows that there exists a multi- $\mathcal{P}$-contact embedding germ $C^{\prime \prime}=\left(C_{1}^{\prime \prime}, \ldots, C_{m}^{\prime \prime}\right):\left(J^{1}\left(\mathbb{R} \times \mathbb{R}^{n}, \mathbb{R}\right)^{(m)}, 0\right) \rightarrow J^{1}\left(\mathbb{R} \times \mathbb{R}^{n}, \mathbb{R}\right)^{(m)}$ such that $\mathcal{L}$ and $\mathcal{L}^{\prime \prime}:=\left(\left.C_{1}^{\prime \prime}\right|_{\tilde{\mathbb{L}}^{r_{1}}}, \ldots,\left.C_{m}^{\prime \prime}\right|_{\tilde{\mathbb{L}}^{r_{m}}}\right)$ are $\mathcal{P}_{(m)}$-Legendrian equivalent and $j_{0}^{l} C_{i}^{\prime \prime}(0)=j_{0}^{l} \tilde{C}_{i x}^{\prime}(0)$. Since $\mathcal{L}$ is infinitesimally stable, it follows that $\mathcal{L}$ is $(n+3)$-determined by Theorem 5.3. Therefore we have that $\mathcal{L}^{\prime \prime}$ is also $(n+3)$-determined. Then $\mathcal{L}^{\prime \prime}$ and $\left(\left.\left(\tilde{C}_{1}^{\prime}\right)_{x_{1}}\right|_{\mathbb{L}^{r_{1}}}, \ldots,\left.\left(\tilde{C}_{m}^{\prime}\right)_{x_{m}}\right|_{\mathbb{L}^{r_{m}}}\right)$ are $\mathcal{P}$-Legendrian equivalent. This means that $\mathcal{L}$ is stable.

Let $\mathcal{L}=\left(\mathcal{L}_{1}, \ldots, \mathcal{L}_{m}\right)$ be a stable multi-reticular Legendrian unfolding. We say that $\mathcal{L}$ is simple if there exists a representative $\tilde{C} \in C_{T}\left(U, J^{1}\left(\mathbb{R} \times \mathbb{R}^{n}, \mathbb{R}\right)\right)^{(m)}$ of a extension of $\mathcal{L}$ such that $\left\{\tilde{C}_{x} \mid x \in U\right\}$ is covered by finite orbits $\left[C_{1}\right], \ldots,\left[C_{l}\right]$ for some multi-P -contact embedding germs $C_{1}, \ldots, C_{l} \in C_{T}\left(U, J^{1}\left(\mathbb{R} \times \mathbb{R}^{n}, \mathbb{R}\right)\right)^{(m)}$.

Proposition 5.5. Let $\mathcal{L}=\left(\mathcal{L}_{1}, \ldots, \mathcal{L}_{m}\right)$ be a stable multi-reticular Legendrian unfolding. Then $\mathcal{L}$ is simple if and only if all reticular Legendrian unfoldings $\mathcal{L}_{i}$ are simple for $i=1, \ldots, m$. 
In order to classify generic multi-reticular Legendrian unfoldings, we classify stable unfoldings $F=\left(F_{1}, \ldots, F_{m}\right)(n \leq 2, m \geq 2)$ of $f=\left(f_{1}, \ldots, f_{m}\right)$ and $f_{0}=\left(f_{0,1}, \ldots, f_{0, m}\right)$ satisfying the condition: the reticular $(\mathcal{P}-\mathcal{K})_{(m-1)}$-codimension of $\left(f_{1}, \ldots, \check{f}_{i}, \ldots, f_{m}\right)=0$ for any $i$.

Let a stable unfoldings $F=\left(F_{1}, \ldots, F_{m}\right)(n \leq 2, m \geq 2)$ of $f=\left(f_{1}, \ldots, f_{m}\right)$ and $f_{0}=\left(f_{0,1}, \ldots, f_{0, m}\right)$ satisfying the condition be given. Then each $F_{i}$ is a reticular $t$-P $\mathcal{P}-\mathcal{K}$-stable unfolding of $f_{i}$, there exist monomials $\varphi_{i, 1}, \ldots, \varphi_{i, \mu_{i}} \in \mathfrak{M}\left(r_{i} ; k_{i}\right)$ such that they consist a basis of $Q_{f_{0, i}}=\left\langle f_{0, i}, x \frac{\partial f_{0, i}}{\partial x}, \frac{\partial f_{0, i}}{\partial y}\right\rangle_{\mathcal{E}\left(r_{i} ; k_{i}\right)}$ and $\varphi_{i, 1}$ has the maximal degree, and $\varphi_{i, \mu_{i}}=1$. Then we have that $\mu_{1}+\cdots \mu_{m} \leq n+2$. Since $\sum_{i=1}^{m} \mu_{i} \leq n+2 \leq 4$, we have that all $f_{0, i}$ are simple singularities. Therefore $f_{0}$ is stably reticular $\mathcal{K}_{(m)}$-equivalent to one of the multi-germs in the following list:

$n=1$;

$m=1 ; y^{2}, y^{3}, y^{4}, x^{2}, x^{3}, \pm x y+y^{3}$,

$m=2 ;\left(y^{2}, y^{2}\right),\left(y^{2}, y^{3}\right),\left(y^{2}, x^{2}\right)$,

$m=3 ;\left(y^{2}, y^{2}, y^{2}\right)$,

$n=2$;

$m=1 ; y^{2}, y^{3}, y^{4}, y^{5}, y_{1}^{2} \pm y_{2}^{2}, x^{2}, x^{3}, x^{4}, \pm x y+y^{3}, x y+y^{4}, x^{2}+y^{3}$,

$m=2 ;\left(y^{2}, y^{2}\right),\left(y^{2}, y^{3}\right),\left(y^{2}, y^{4}\right),\left(y^{3}, y^{3}\right),\left(y^{2}, x^{2}\right),\left(y^{2}, x^{3}\right),\left(y^{2}, \pm x y+y^{3}\right),\left(x^{2}, x^{2}\right)$, $m=3 ;\left(y^{2}, y^{2}, y^{2}\right),\left(y^{2}, y^{2}, y^{3}\right),\left(y^{2}, y^{2}, x^{2}\right)$,

$m=4 ;\left(y^{2}, y^{2}, y^{2}, y^{2}\right)$.

We construct a reticular $(\mathcal{P}-\mathcal{K})_{(m)}$-versal unfolding for each germ by the usual method. Then the corresponding list is as follows:

$n=1$;

(1) $\left(y^{2}+u_{1,1}, y^{2}+u_{2,1}\right)$

(2) $\left(y^{2}+u_{1,1}, y^{3}+u_{2,1} y+u_{2,2}\right)$

(3) $\left(y^{2}+u_{1,1}, x^{2}+u_{2,1} x+u_{2,2}\right)$

(4) $\left(y^{2}+u_{1,1}, y^{2}+u_{2,1}, y^{2}+u_{3,1}\right)$

$n=2$;

(6) $\left(y^{2}+u_{1,1}, y^{2}+u_{2,1}\right)$

(7) $\left(y^{2}+u_{1,1}, y^{3}+u_{2,1} y+u_{2,2}\right)$

(8) $\left(y^{2}+u_{1,1}, y^{4}+u_{2,1} y^{2}+u_{2,2} y+u_{2,3}\right)$

(9) $\left(y^{3}+u_{1,1} y+u_{1,2}, y^{3}+u_{2,1} y+u_{2,2}\right)$

(10) $\left(y^{2}+u_{1,1}, x^{2}+u_{2,1} x+u_{2,2}\right)$

(11) $\left(y^{2}+u_{1,1}, x^{3}+u_{2,1} x^{2}+u_{2,2} x+u_{2,3}\right)$

(12) $\left(y^{2}+u_{1,1}, \pm x y+y^{3}+u_{2,1} y^{2}+u_{2,2} y+u_{2,3}\right)$

(13) $\left(x^{2}+u_{1,1} x+u_{1,2}, x^{2}+u_{2,1} x+u_{2,2}\right)$

(14) $\left(y^{2}+u_{1,1}, y^{2}+u_{2,1}, y^{2}+u_{3,1}\right)$

(15) $\left(y^{2}+u_{1,1}, y^{2}+u_{2,1}, y^{3}+u_{3,1} y+u_{3,2}\right)$

(16) $\left(y^{2}+u_{1,1}, y^{2}+u_{2,1}, x^{2}+u_{3,1} x+u_{3,2}\right)$

(17) $\left(y^{2}+u_{1,1}, y^{2}+u_{2,1}, y^{2}+u_{3,1}, y^{2}+u_{4,1}\right)$.

In the case that the reticular $(\mathcal{P}-\mathcal{K})_{(m)}$-codimension of $f=0$, the map germ $F$ is stably reticular $t$ - $(\mathcal{P}-\mathcal{K})_{(m)}$-equivalent to $G=\left(G_{1}, \ldots, G_{m}\right)$, where $G_{i}(x, y, t, q, z)=$ $f_{0, i}(x, y)+u_{i, 1} \varphi_{1}(x, y)+\cdots+u_{i, \mu_{i}} \varphi_{i, \mu_{i}}(x, y)-z$ for $i=1, \ldots, m-1, G_{m}(x, y, t, q, z)=$ $f_{0, i}(x, y)+u_{m, 1} \varphi_{1}(x, y)+\cdots+u_{m, \mu_{m-1}} \varphi_{m, \mu_{m-1}}(x, y)-z$, and $\left(q_{1}, \ldots, q_{n}, z\right)=$ $\left(u_{1,1}, \ldots, u_{1, \mu_{1}}, \ldots, u_{m, 1}, \ldots, u_{m, \mu_{m-1}}, u_{1}, \ldots, u_{\mu}, z\right)$.

In the case that the reticular $(\mathcal{P}-\mathcal{K})_{(m)}$-codimension of $f=1, F$ is stably reticular 
$t$ - $(\mathcal{P}-\mathcal{K})_{(m)}$-equivalent to $\left(F_{1}^{\prime}, \ldots, F_{m}^{\prime}\right)$, where

(1) $F_{1}^{\prime}=f_{0,1}(x, y)+(t+a(q, z)) \varphi_{1}(x, y)+u_{1,1} \varphi_{2}(x, y) \cdots+u_{1, \mu_{1}-1} \varphi_{1, \mu_{1}}(x, y)-z$,

(2) $F_{i}^{\prime}=f_{0, i}(x, y)+u_{i, 1} \varphi_{1}(x, y)+\cdots+u_{i, \mu_{i}} \varphi_{i, \mu_{i}}(x, y)-z$ for $1<i<m$,

(3) $F_{m}^{\prime}=f_{0, m}(x, y)+u_{m, 1} \varphi_{m, 1}(x, y)+\cdots+u_{m, \mu_{m}-1} \varphi_{m, \mu_{m}-1}(x, y)-z$,

(4) $\left(q_{1}, \ldots, q_{n}, z\right)=\left(u_{1,1}, \ldots, u_{1, \mu_{1}-1}, u_{2,1}, \ldots, u_{2, \mu_{2}}, \ldots, u_{m, 1}, \ldots, u_{m, \mu_{m-1}}\right.$, $\left.u_{1}, \ldots, u_{\mu}, z\right)$,

(5) $\frac{\partial a}{\partial u_{1, j}}(0)=0$ for $j=1, \ldots, \mu_{1}-1, \frac{\partial a}{\partial z}(0)=0$, and $\left(\frac{\partial^{2} a}{\partial u_{i} \partial u_{j}}(0)\right)_{i, j=1, \ldots, \mu}$ is non-degenerate.

We denote the linear part of $a$ by $v=v_{2}+\cdots+v_{m}$, where $v_{i}$ depends only on $u_{i, 1}, \ldots, u_{i, \mu_{i}}$. Then we may reduce $a$ to

$$
a=t \pm u_{2,1} \pm \cdots \pm u_{m, 1} \pm u_{1}^{2} \pm \cdots u_{\mu}^{2}
$$

Then $F$ is stably reticular $t-(\mathcal{P}-\mathcal{K})_{(m)}$-equivalent to one of the following list: $n=1$;

$m=2$;

${ }^{0}\left({ }^{0} A_{1}{ }^{0} A_{1}\right): \quad\left(y^{2}+q-z, y^{2}-z\right) ;$

${ }^{1}\left({ }^{0} A_{1}^{0} A_{1}\right): \quad\left(y^{2}+t \pm q^{2}-z, y^{2}-z\right)$;

${ }^{1}\left({ }^{0} A_{1}{ }^{0} A_{2}\right):\left(y^{2}+t \pm q-z, y^{3}+q y-z\right) ;$

${ }^{1}\left({ }^{0} A_{1}{ }^{0} B_{2}\right):\left(y^{2}+t \pm q-z, x^{2}+q x-z\right)$.

$m=3$;

${ }^{1}\left({ }^{0} A_{1}{ }^{0} A_{1}^{0} A_{1}\right):\left(y^{2}+t-z, y^{2}+q-z, y^{2}-q-z\right)$.

$n=2$;

$m=2$;

${ }^{1}\left({ }^{0} A_{1}{ }^{0} A_{1}\right): \quad\left(y^{2}+t \pm q_{1}^{2} \pm q_{2}^{2}-z, y^{2}-z\right) ;$

${ }^{0}\left({ }^{0} A_{1}{ }^{0} A_{2}\right): \quad\left(y^{2}+t \pm q_{1}-z, y^{3}+q_{1} y-q_{2}-z\right) ;$

${ }^{1}\left({ }^{0} A_{1}{ }^{0} A_{2}\right): \quad\left(y^{2}+t \pm q_{1} \pm q_{2}^{2}-z, y^{3}+q_{1} y-z\right) ;$

${ }^{0}\left({ }^{0} A_{1}{ }^{0} B_{2}\right): \quad\left(y^{2}+q_{1}-z, x^{2}+q_{2} x-z\right) ;$

${ }^{1}\left({ }^{0} A_{1}{ }^{0} A_{3}\right): \quad\left(y^{2}+t \pm q_{1}-z, y^{4}+q_{1} y^{2}+q_{2} y-z\right) ;$

${ }^{1}\left({ }^{0} A_{2}{ }^{0} A_{2}\right): \quad\left(y^{3}+\left(t \pm q_{1}\right) y+q_{2}-z, y^{3}+q_{1} y-z\right) ;$

${ }^{1}\left({ }^{0} A_{1}^{0} B_{2}\right): \quad\left(y^{2}+t \pm q_{1} \pm q_{2}^{2}-z, x^{2}+q_{1} x-z\right) ;$

${ }^{1}\left({ }^{0} A_{1} B_{3}\right): \quad\left(y^{2}+t \pm q_{1}-z, x^{3}+q_{1} x^{2}+q_{2} x-z\right) ;$

${ }^{1}\left({ }^{0} A_{1}{ }^{0} C_{3}^{ \pm}\right): \quad\left(y^{2}+t+q_{1}-z, \pm x y+y^{3}+q_{1} y^{2}+q_{2} y-z\right)$;

${ }^{1}\left({ }^{0} B_{2}{ }^{0} B_{2}\right):\left(x^{2}+\left(t \pm q_{1}\right) x+q_{2}-z, x^{2}+q_{1} x-z\right)$.

$m=3$;

${ }^{0}\left({ }^{0} A_{1}{ }^{0} A_{1}{ }^{0} A_{1}\right): \quad\left(y^{2}+q_{1}-z, y^{2}+q_{2}-z, y^{2}-z\right) ;$

${ }^{1}\left({ }^{0} A_{1}^{0} A_{1}^{0} A_{1}\right): \quad\left(y^{2}+t \pm q_{1} \pm q_{2}^{2}-z, y^{2}+q_{1}-z, y^{2}-z\right) ;$

${ }^{1}\left({ }^{0} A_{1}^{0} A_{1}^{0} A_{2}\right): \quad\left(y^{2}+t \pm q_{1}-z, y^{2}-z, y^{3}+q_{1} y+q_{2}-z\right)$;

${ }^{1}\left({ }^{0} A_{1}{ }^{0} A_{1}{ }^{0} B_{2}\right):\left(y^{2}+t \pm q_{1}-z, y^{2}-z, x^{2}+q_{1} x+q_{2}-z\right)$.

$m=4$;

${ }^{1}\left({ }^{0} A_{1}^{0} A_{1}^{0} A_{1}^{0} A_{1}\right):\left(y^{2}+t \pm q_{1} \pm q_{2}-z, y^{2}+q_{1}-z, y^{2}+q_{2}-z, y^{2}-z\right)$.

THEOREM 5.6. Let $r_{i}=0$ or 1 for $i=1, \ldots, m$ and $n \leq 2$. Let $U$ be $a$ neighborhood of 0 in $J^{1}\left(\mathbb{R} \times \mathbb{R}^{n}, \mathbb{R}\right)$. Then there exists a residual set $\bar{O} \subset C_{T}^{\Theta}\left(U, J^{1}(\mathbb{R} \times\right.$ $\left.\left.\mathbb{R}^{n}, \mathbb{R}\right)\right)^{(m)}$ such that for any $\tilde{C}=\left(\tilde{C}_{1}, \ldots, \tilde{C_{m}}\right) \in O$ and $x=\left(x_{1}, \ldots, x_{m}\right) \in U^{(m)}$, the multi-reticular Legendrian unfolding $\left(\left.\left(\tilde{C_{1}}\right)_{x_{1}}\right|_{\mathbb{L}^{r_{1}}}, \ldots,\left(\tilde{C_{m}}\right)_{x_{m}} \mid \mathbb{L}^{r_{m}}\right)$ is stable and have 
a generating family which is stably reticular $t-(\mathcal{P}-\mathcal{K})_{(m)}$-equivalent for one of the types in the above list.

Proof. Let $X_{i}=\left(X_{i, 1}, \cdots, X_{i, m}\right)(i=1, \ldots, s)$ be all simple singularities with $\sum_{j=1}^{m} r$ - $\mathcal{K}$-codim $X_{i, j} \leq n+2$, that is each $X_{i, j}$ is one of simple singularities $A, B, C$. Let $F_{X_{i}}$ be $\mathcal{P}_{(m)}$ - $C$-non-degenerate map germ which is unfolding of $X_{i}$ for $i=1, \ldots, s$. We choose an extension $C_{\left({ }^{0} X_{i}\right)}$ and $C_{\left({ }^{1} X_{i}\right)}$ of multi-reticular Legendrian unfoldings with multi-generating families $F_{\left({ }^{0} X_{i}\right)}$ and $\left.F_{(1} X_{i}\right)$ respectively. Let $l>16$. We define that

$$
\begin{aligned}
O^{\prime}=\left\{\tilde{C} \in C_{T}^{\Theta}(U,\right. & \left.J^{1}\left(\mathbb{R} \times \mathbb{R}^{n}, \mathbb{R}\right)\right)^{(m)} \mid j_{0}^{l} \tilde{C} \text { is transversal to } \\
& {\left[\left(j^{l} C_{\left(j X_{i}\right)}(0)\right] \text { for all } i=1, \ldots, s \text { and } j=0,1\right\} . }
\end{aligned}
$$

Let $X_{i}^{\prime}=\left(X_{i, 1}, \ldots, X_{i, m-1}\right)\left(i=1, \ldots, s^{\prime}\right)$ be all simple singularities with $\sum_{j=1}^{m-1} r-\mathcal{K}$ $\operatorname{codim} X_{i, j} \leq n+1$. We choose $C_{\left({ }^{0} X_{i}^{\prime}\right)} \in C_{T}^{\Theta}\left(U, J^{1}\left(\mathbb{R} \times \mathbb{R}^{n}, \mathbb{R}\right)\right)^{(m-1)}$ by an analogous way. Then we define that

$$
\begin{array}{r}
O^{\prime \prime}=\left\{\tilde{C} \in C_{T}^{\Theta}\left(U, J^{1}\left(\mathbb{R} \times \mathbb{R}^{n}, \mathbb{R}\right)\right)^{(m)} \mid\left(j_{0}^{l} \tilde{C}_{1}, \ldots, j_{0}^{l} \tilde{C_{j}}, \ldots, j_{0}^{l} \tilde{C_{m}}\right)\right. \text { is } \\
\text { transversal to }\left[\left(j^{l} C_{\left({ }^{0} X_{i}^{\prime}\right)}(0)\right] \text { for all } i=1 \cdots, s^{\prime}\right\} .
\end{array}
$$

Then $O^{\prime}$ and $O^{\prime \prime}$ are residual sets. We set

$$
Y=\left\{j^{l} C(0) \in t C^{l}(n, m) \mid \text { the codimension of }\left[j^{l} C(0)\right]>2 n+4\right\} .
$$

Then $Y$ is an algebraic set in $t C^{l}(n, m)$. Therefore we can define that

$$
O^{\prime \prime \prime}=\left\{\tilde{C} \in C_{T}^{\Theta}\left(U, J^{1}\left(\mathbb{R} \times \mathbb{R}^{n}, \mathbb{R}\right)\right)^{(m)} \mid j_{0}^{l} \tilde{C} \text { is transversal to } Y\right\} .
$$

Then $Y$ has codimension $>2 n+4$ because all $\mathcal{P}_{(m)}$-contact embedding germ with $j^{l} C(0) \in Y$ adjoin to the above list which are simple. Therefore

$$
O^{\prime \prime \prime}=\left\{\tilde{C} \in C_{T}^{\Theta}\left(U, J^{1}\left(\mathbb{R} \times \mathbb{R}^{n}, \mathbb{R}\right)\right)^{(m)} \mid j_{0}^{l} \tilde{C}\left(U^{(m)}\right) \cap Y=\emptyset\right\} .
$$

Then the set $O=O^{\prime} \cap O^{\prime \prime} \cap O^{\prime \prime \prime}$ has the required condition.

6. Classification for the cases $r \geq 2$. In order to classify generic bifurcations of wavefronts in the case $r \geq 2$, our methods do not work well. Since the most simple singularity $f\left(x_{1}, x_{2}\right)=x_{1}^{2}+a x_{1} x_{2} \pm x_{2}^{2}\left(B_{2,2}^{ \pm, a}\right)$ have the reticular $\mathcal{P}$ - $\mathcal{K}$-codimension 4 and one modality. By the classification list in [6, p.127], we have that all singularities for $r \geq 2$ are adjacent to $B_{2,2}^{ \pm, a}$ (cf., [5, p.593]). This means that all of them have modalities and hence Theorem 5.6 does not work well. We need other equivalence relations among reticular Legendrian unfoldings for the cases $r \geq 2$.

\section{REFERENCES}

[1] V. I. Arnold, S. M. Gusein-Zade, and A. N. Varchenko, Singularities of differential maps I, Birkhauser, 1985.

[2] S. Izumiya and G. T. Kossionis, Semi-local classification of geometric singularities for hamilton-jacobi equations, Journal of Differential equation, 118 (1995), pp. 166-193.

[3] K. JÄNICH, Caustics and catastrophes, Mathematische Annalen, 209:2 (1974), pp. 161-180.

[4] I. G. ScherbaK, Boundary fronts and caustics and their metamorphoses, Singularities (Lille 1991): pp. 363-373, London Math. Soc. Lecture Note 201, Cambridge Univ. Press, Cambridge, 1994. 
[5] T. TsukADA, Reticular lagrangian singularities, The Asian J. of Math., 1:3 (1997), pp. 572-622.

[6] T. TsukAdA, Reticular legendrian singularities, The Asian J. of Math., 5:1 (2001), pp. 109-127.

[7] T. TsukADA, A generic classification of function germs with respect to the reticular $t-\mathcal{P}-\mathcal{K}$ equivalence, Hokkaido Math. J., 38:1 (2009), pp. 177-203.

[8] T. Tsukada, Genericity of caustics and wavefronts on an r-corner, Asian J. Math., 14:3 (2010), pp. 335-358.

[9] G. Wassermann, Stability of unfolding in space and time, Acta Mathematica, 135:1 (1975), pp. $57-128$.

[10] V. M .ZAKAlYUkin, Reconstructions of fronts and caustics depending on a parameter, and versality of mappings, J. Soviet Math., 27:3 (1984), pp. 2713-2735.

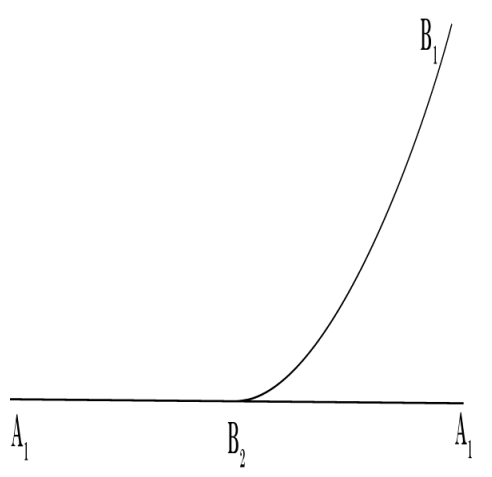

FIG. 3. ${ }^{0} B_{2}$

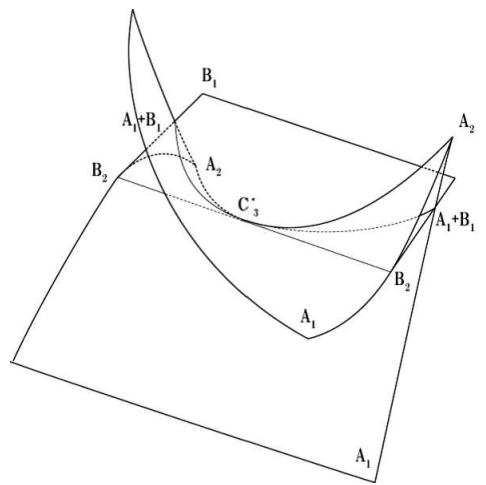

FIG. $5 .{ }^{0} C_{3}^{-}$

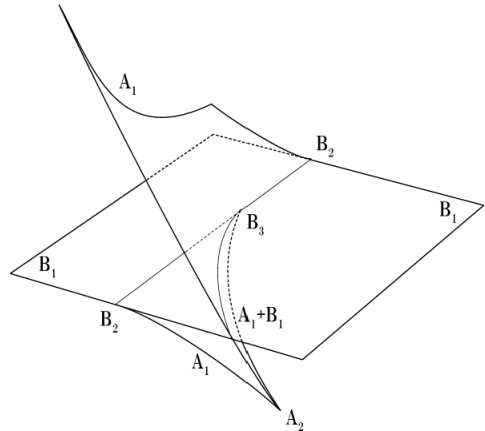

FIG. $4 .{ }^{0} B_{3}$

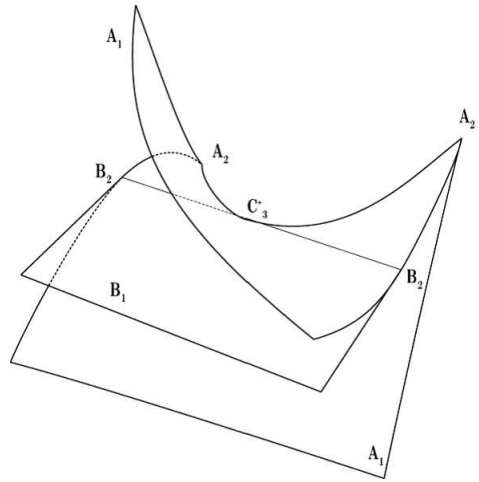

FIG. $6 .{ }^{0} C_{3}^{+}$ 

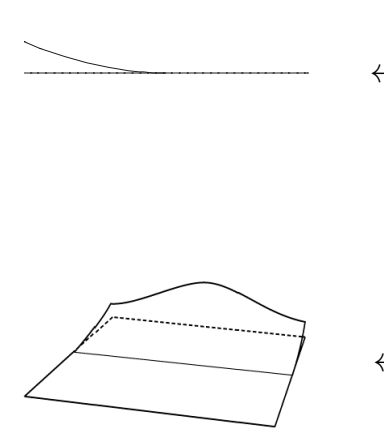

$\leftrightarrow$
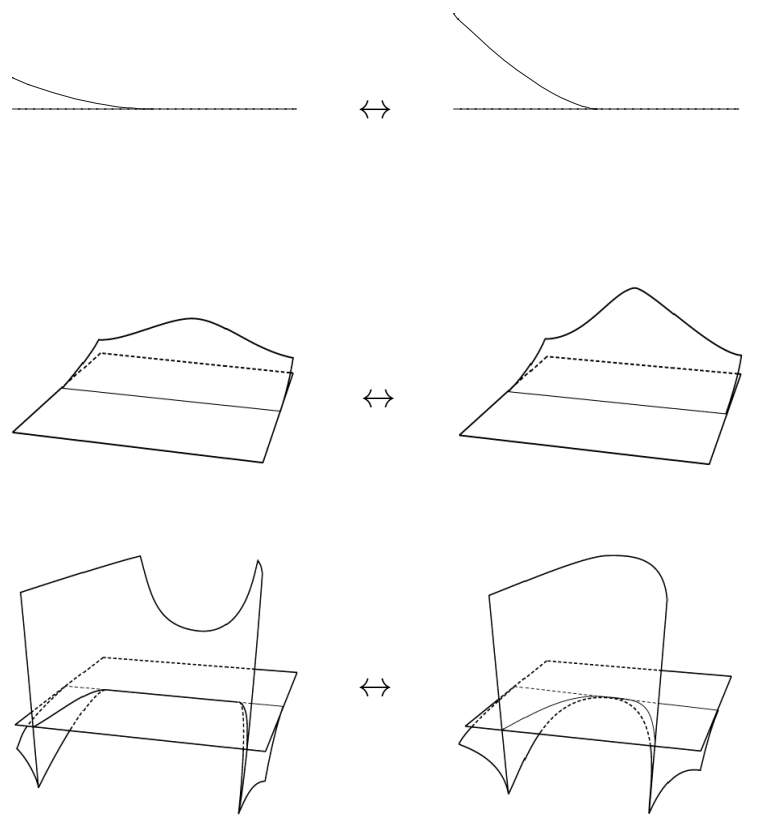

$\leftrightarrow$

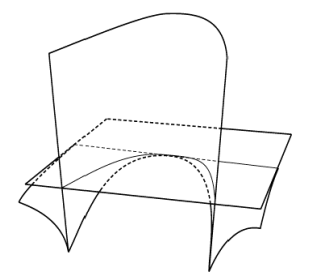

FIG. $7 .{ }^{1} B_{3}$

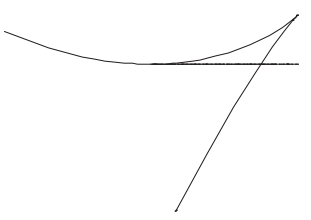

$\leftrightarrow$

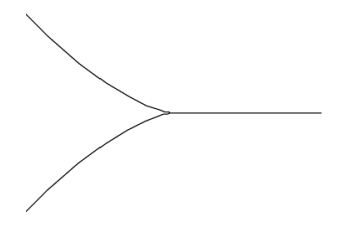

$\leftrightarrow$
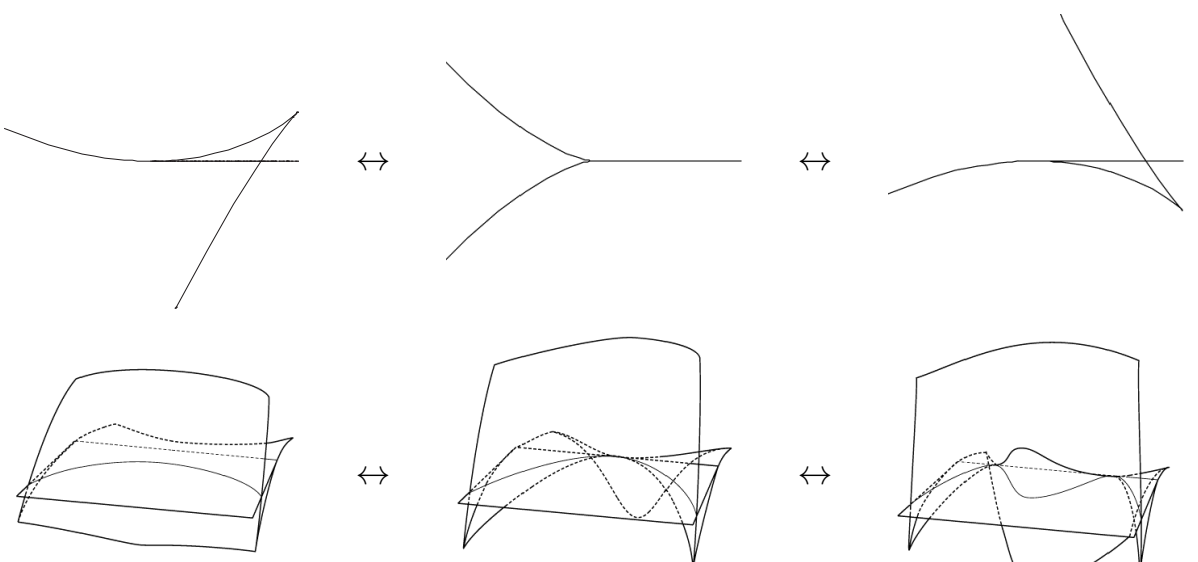

$\leftrightarrow$

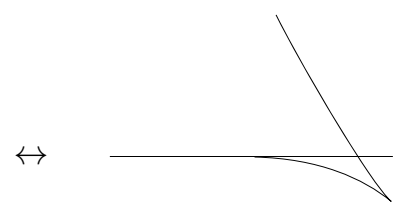

$\leftrightarrow$
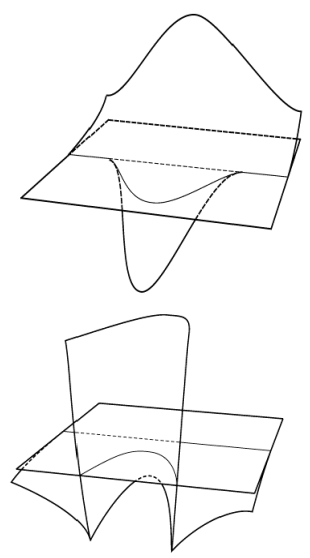
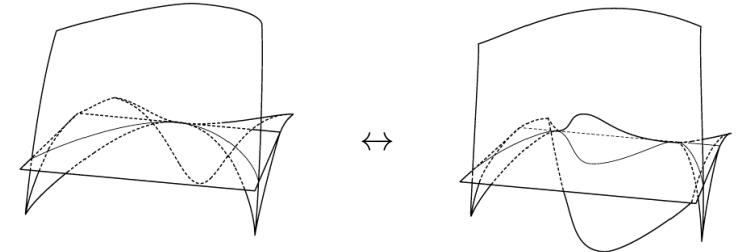

FIG. $8 .{ }^{1} C_{3}^{-}$ 


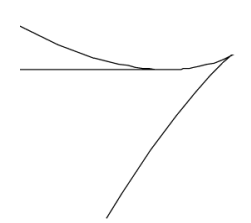

$\leftrightarrow$
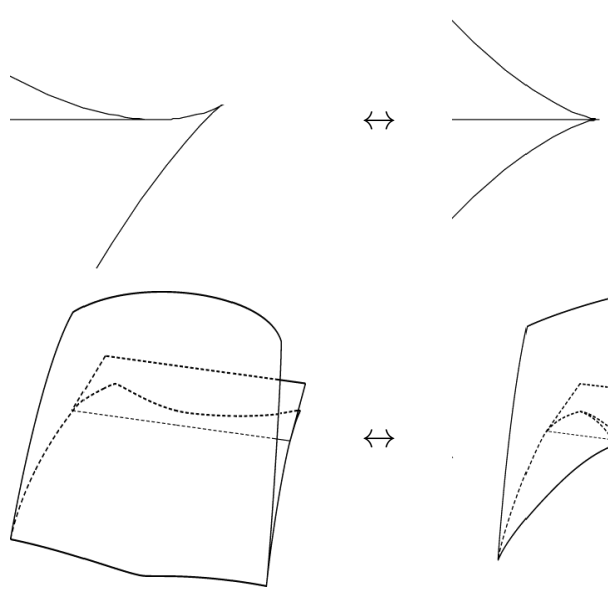

$\leftrightarrow$

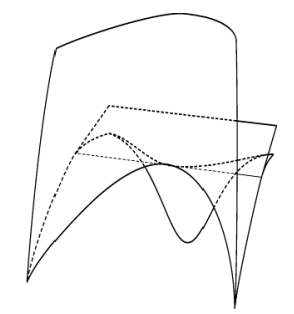

FIG. $9 .{ }^{1} C_{3}^{+}$

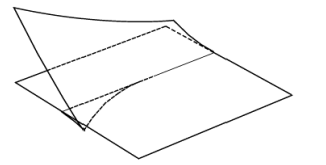

$\leftrightarrow$

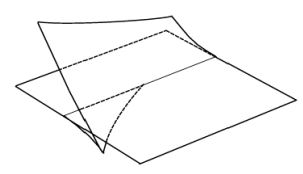

$\leftrightarrow$

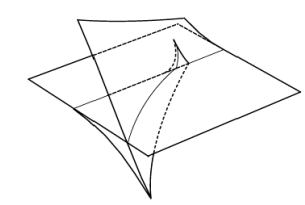

FIG. 10. ${ }^{1} B_{4}$

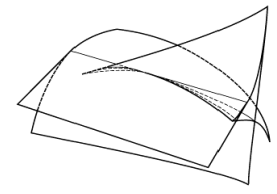

$\leftrightarrow$

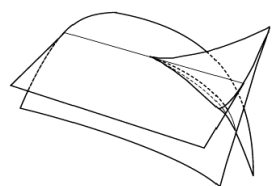

$\leftrightarrow$

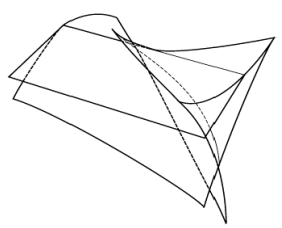

FIG. $11 .{ }^{1} C_{4}$ 

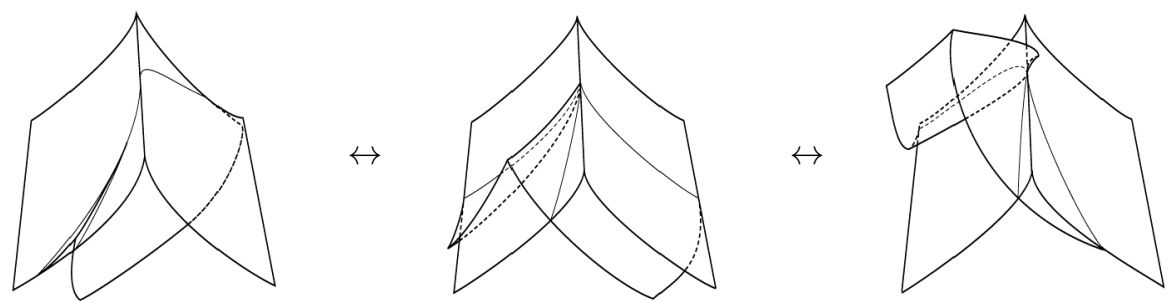

FIG. $12 .{ }^{1} F_{4}$

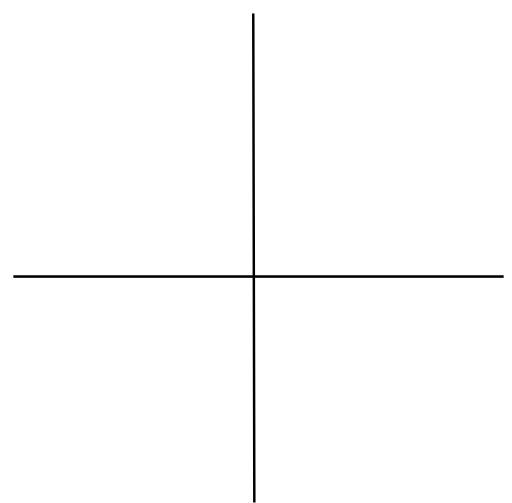

FIG. $13 .{ }^{0}\left({ }^{0} A_{1}{ }^{0} A_{1}\right)$

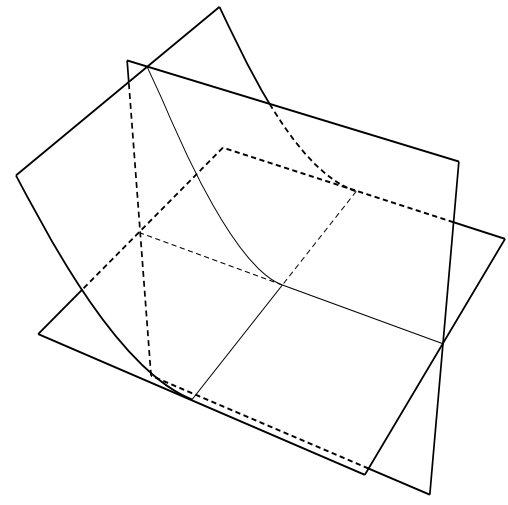

FIG. $15 .{ }^{0}\left({ }^{0} A_{1}{ }^{0} B_{2}\right)$

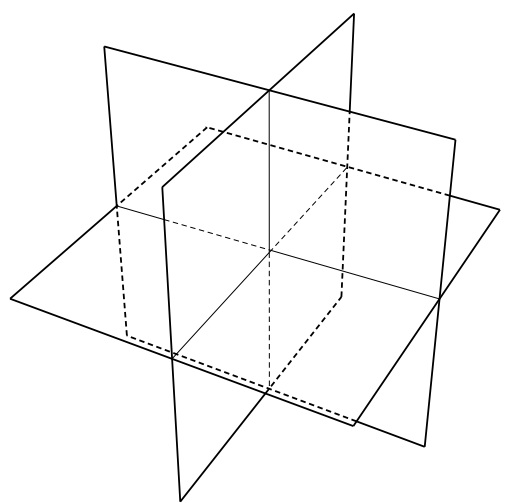

FIG. $14 .{ }^{0}\left({ }^{0} A_{1}{ }^{0} A_{1}{ }^{0} A_{1}\right)$

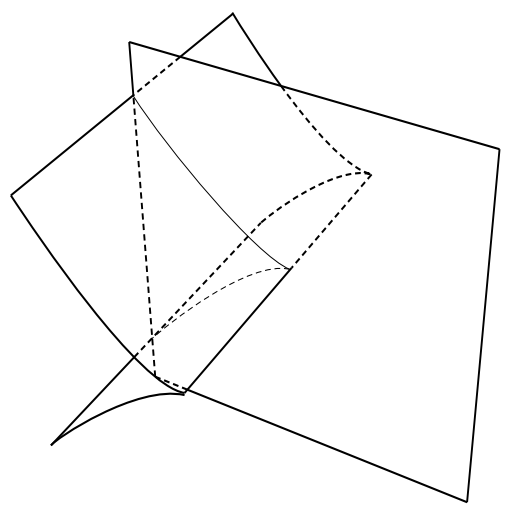

FIG. $16 .{ }^{0}\left({ }^{0} A_{1}{ }^{0} A_{2}\right)$ 

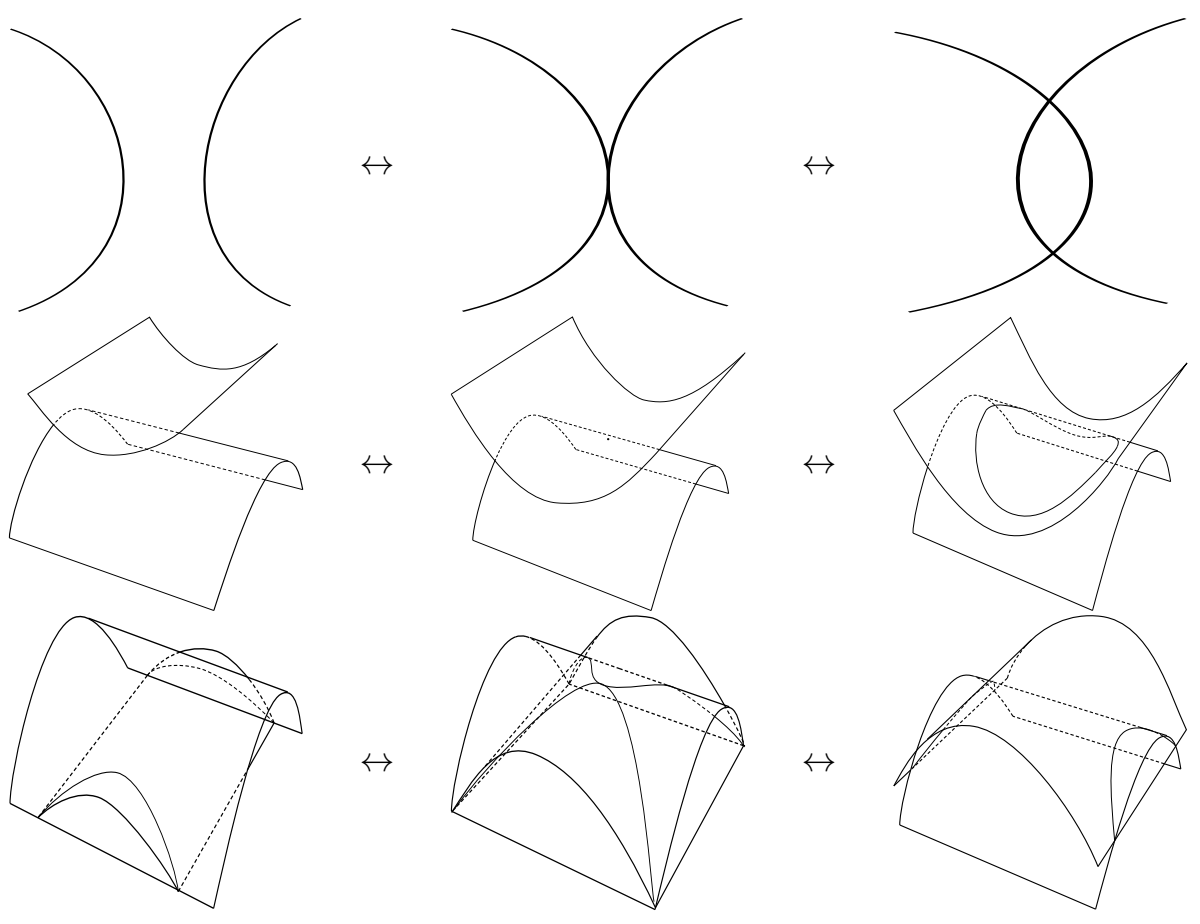

FIG. $17 .{ }^{1}\left({ }^{0} A_{1}{ }^{0} A_{1}\right)$
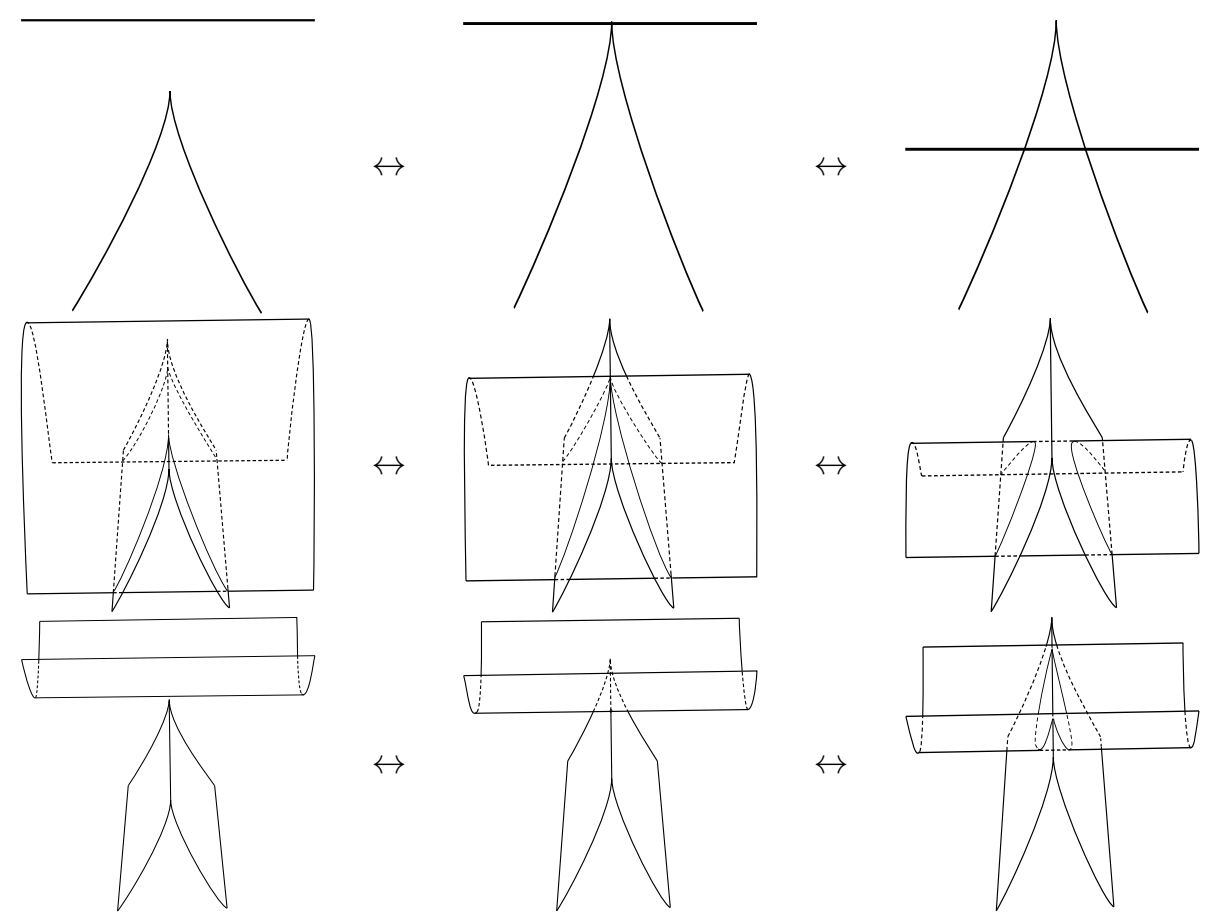

FIG. $18 .{ }^{1}\left({ }^{0} A_{1}{ }^{0} A_{2}\right)$

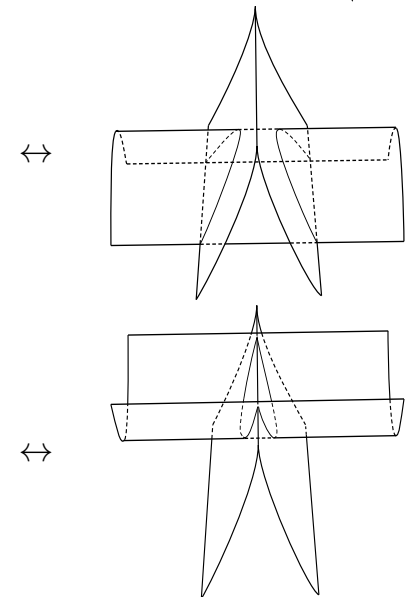



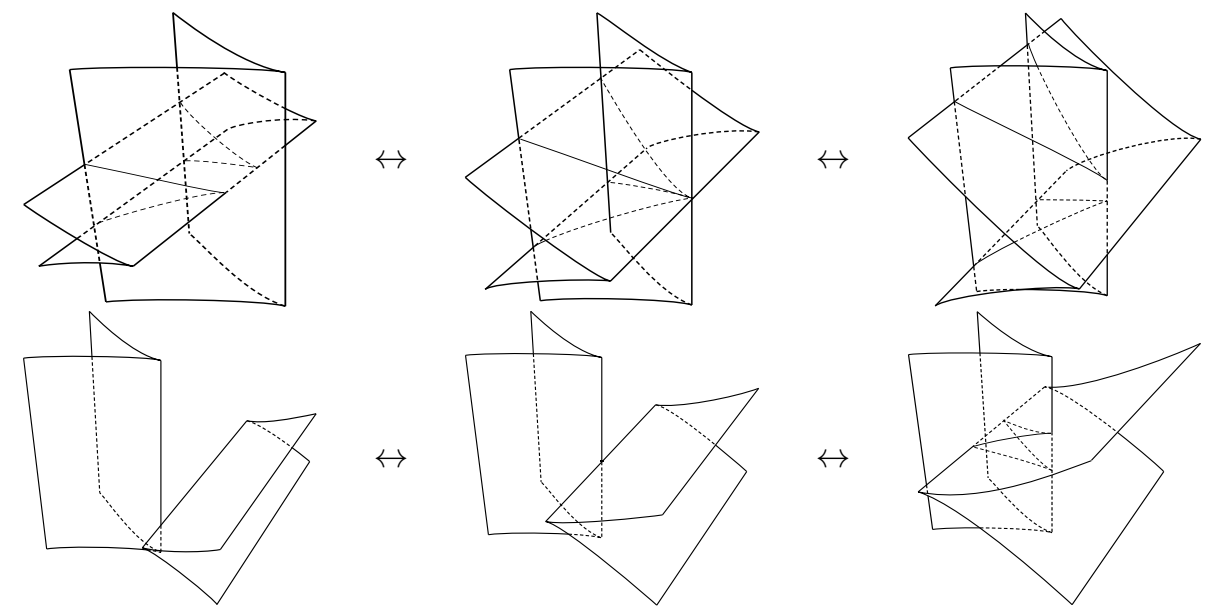

FIG. 19. ${ }^{1}\left({ }^{0} A_{2}{ }^{0} A_{2}\right)$
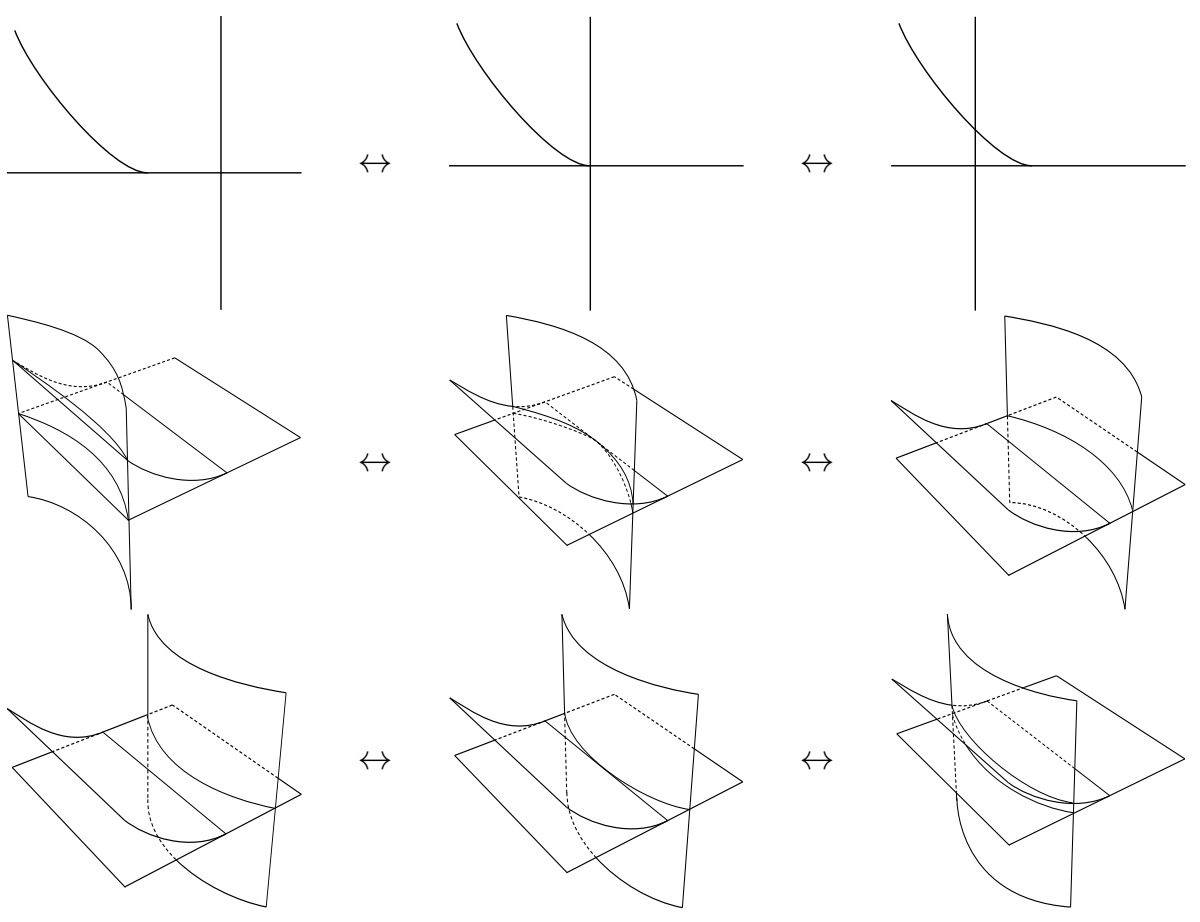

FIG. 20. ${ }^{1}\left({ }^{0} A_{1}{ }^{0} B_{2}\right)$
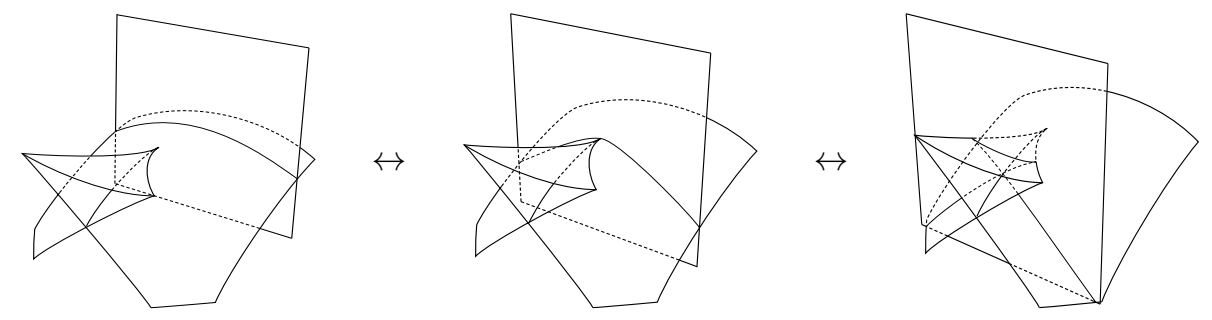

FIG. $21 .{ }^{1}\left({ }^{0} A_{1}{ }^{0} A_{3}\right)$ 

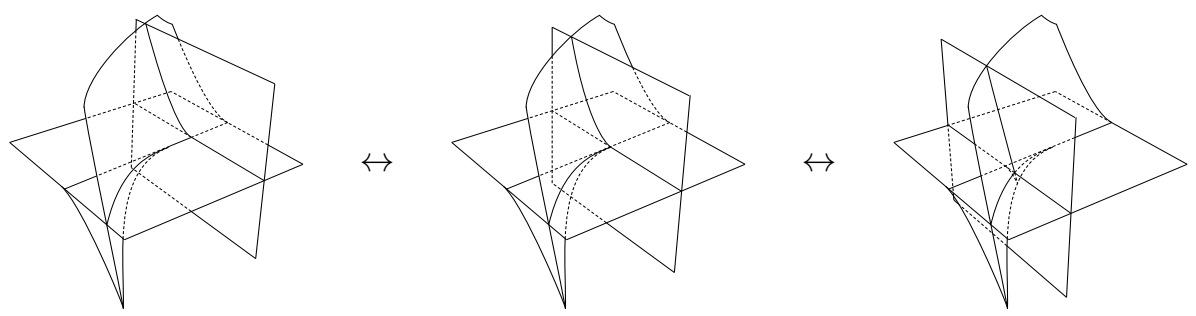

Fig. 22. ${ }^{1}\left({ }^{0} A_{1}{ }^{0} B_{3}\right)$
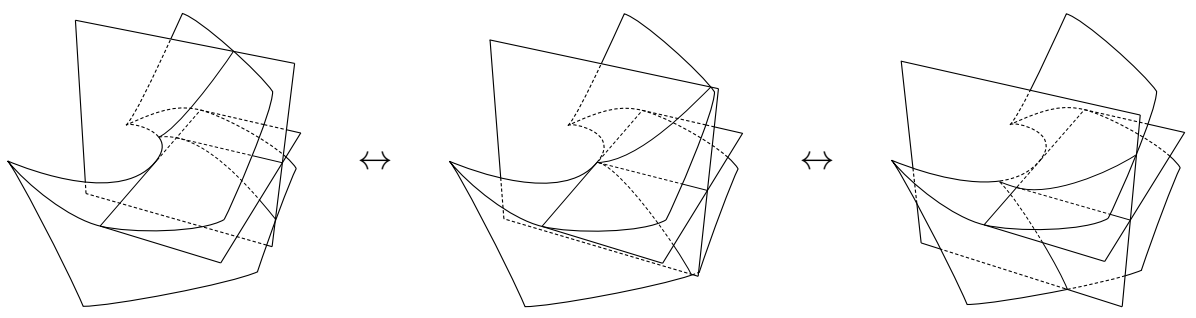

FIG. 23. ${ }^{1}\left({ }^{0} A_{1}{ }^{0} C_{3}^{+}\right)$
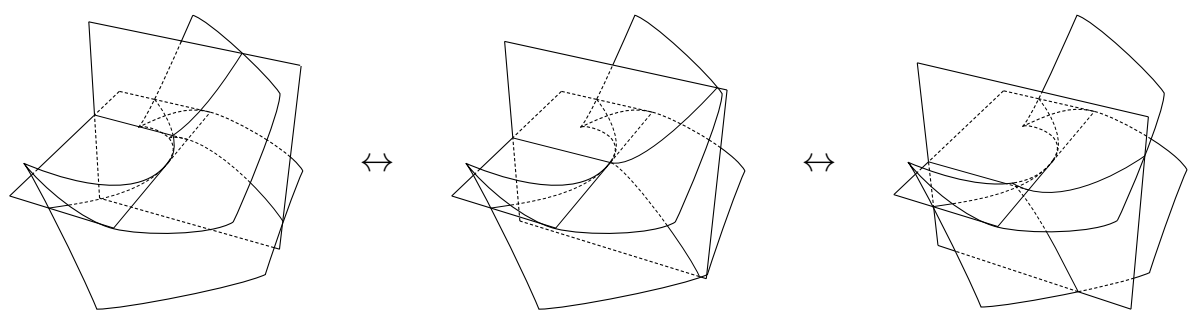

FIG. $24 .{ }^{1}\left({ }^{0} A_{1}{ }^{0} C_{3}^{-}\right)$
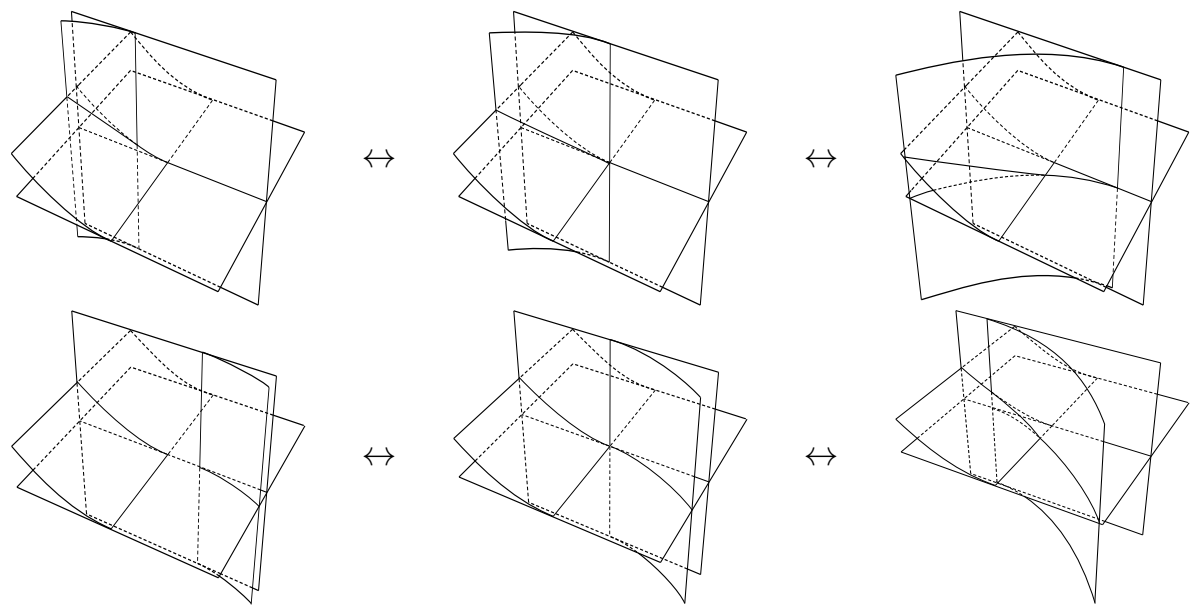

FIG. $25 .{ }^{1}\left({ }^{0} B_{2}{ }^{0} B_{2}\right)$ 

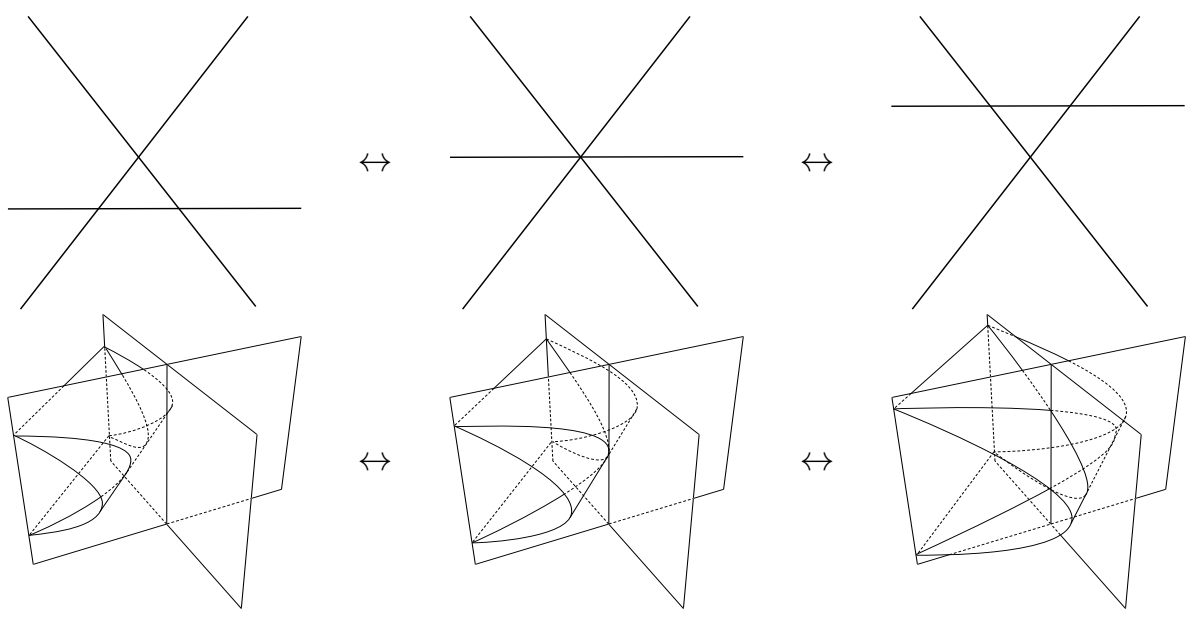

FIG. 26. ${ }^{1}\left({ }^{0} A_{1}{ }^{0} A_{1}{ }^{0} A_{1}\right)$
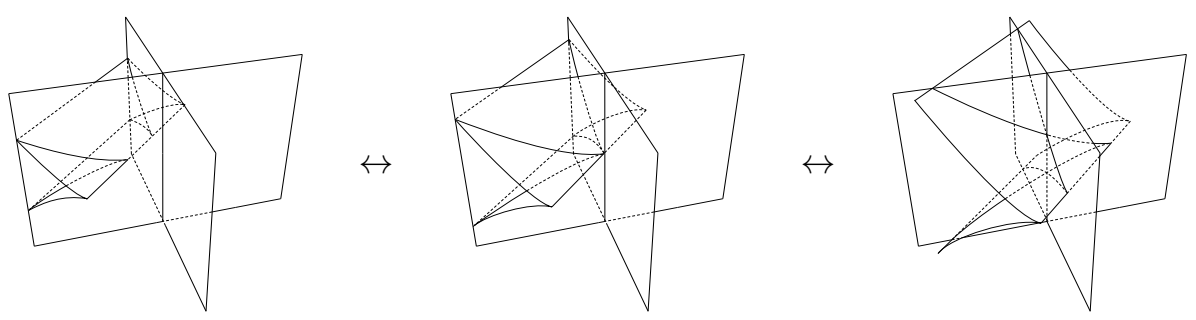

FIG. $27 .{ }^{1}\left({ }^{0} A_{1}{ }^{0} A_{1}{ }^{0} A_{2}\right)$
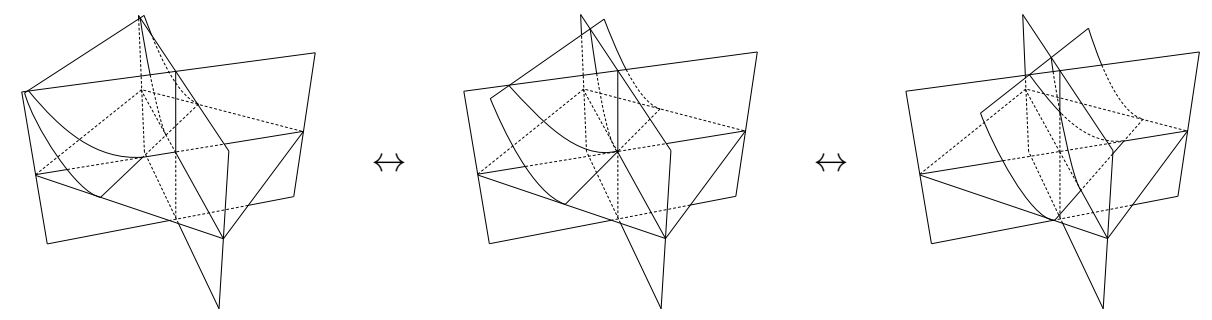

FIG. 28. ${ }^{1}\left({ }^{0} A_{1}{ }^{0} A_{1}{ }^{0} B_{2}\right)$
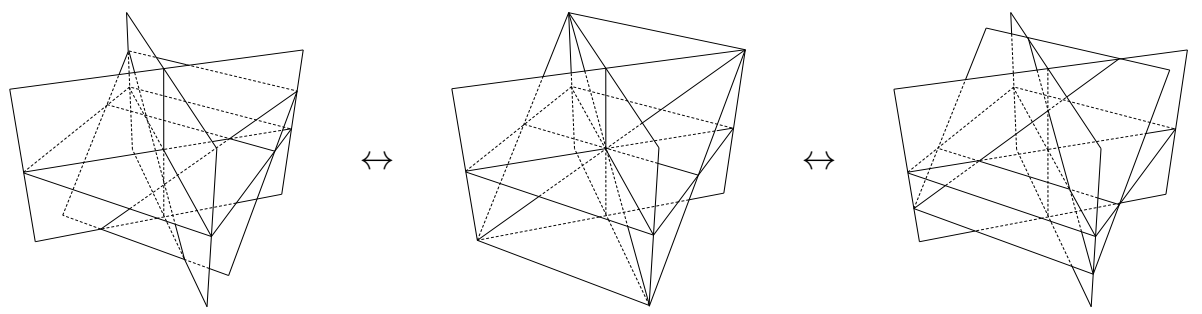

FIG. 29. ${ }^{1}\left({ }^{0} A_{1}{ }^{0} A_{1}{ }^{0} A_{1}{ }^{0} A_{1}\right)$ 